\title{
Complexities of the glomerular basement membrane
}

DOI:

10.1038/s41581-020-0329-y

\section{Document Version}

Accepted author manuscript

Link to publication record in Manchester Research Explorer

\section{Citation for published version (APA):}

Naylor, R. W., Morais, M. R. P. T., \& Lennon, R. (2021). Complexities of the glomerular basement membrane. Nature Reviews Nephrology, 17(2), 112-127. https://doi.org/10.1038/s41581-020-0329-y

\section{Published in:}

Nature Reviews Nephrology

\section{Citing this paper}

Please note that where the full-text provided on Manchester Research Explorer is the Author Accepted Manuscript or Proof version this may differ from the final Published version. If citing, it is advised that you check and use the publisher's definitive version.

\section{General rights}

Copyright and moral rights for the publications made accessible in the Research Explorer are retained by the authors and/or other copyright owners and it is a condition of accessing publications that users recognise and abide by the legal requirements associated with these rights.

\section{Takedown policy}

If you believe that this document breaches copyright please refer to the University of Manchester's Takedown Procedures [http://man.ac.uk/04Y6Bo] or contact uml.scholarlycommunications@manchester.ac.uk providing relevant details, so we can investigate your claim.

\section{OPEN ACCESS}




\title{
Complexities of the glomerular basement membrane
}

Richard W. Naylor ${ }^{1}$, Mychel R. P. T. Morais ${ }^{1,2}$ and Rachel Lennon ${ }^{1,3+}$

1. Wellcome Centre for Cell-Matrix Research, Division of Cell-Matrix Biology and Regenerative Medicine, School of Biological Sciences, Faculty of Biology Medicine and Health, The University of Manchester, Manchester Academic Health Science Centre, Manchester, UK.

2. Laboratory of Reproduction and Extracellular Matrix Biology, Institute of Biomedical Sciences, University of São Paulo, São Paulo, Brazil.

3. Department of Paediatric Nephrology, Royal Manchester Children's Hospital, Manchester University Hospitals NHS Foundation Trust, Manchester Academic Health Science Centre, Manchester, UK.

\section{†Email: Rachel.Lennon@manchester.ac.uk}

\begin{abstract}
The glomerular basement membrane (GBM) is a key component of the glomerular capillary wall and is essential for kidney filtration. Major components of the GBM include laminins, collagen IV, nidogens and heparan sulphate proteoglycans. In addition, the GBM harbours a number of other structural and regulatory components and provides a reservoir for growth factors. New technologies have improved our ability to study the composition and assembly of basement membranes. We now know that the GBM is a complex macromolecular structure that undergoes key transitions during glomerular development. Defects in GBM components are associated with a range of hereditary human diseases such as Alport syndrome, which is caused by defects in the genes COL4A3, COL4A4 and COL4A5 and Pierson syndrome, which is caused by variants in $L A M B 2$. In addition, the GBM is affected by acquired autoimmune disorders and metabolic disease such as diabetes mellitus. Current treatment for diseases associated with GBM involvement aims to reduce intraglomerular pressure and to treat the underlying cause where possible. As our understanding about the maintenance and turnover of the GBM improves, therapies to replace GBM components or to stimulate GBM repair could translate into new therapies for patients with GBM-associated disease.
\end{abstract}




\section{Introduction}

Basement membranes are condensed regions of extracellular matrix (ECM) that underlie continuous sheets of cells. They are found in and around most tissues; however, the full scope of their function is unknown. The evolutionary appearance of basement membranes coincided with the emergence of metazoa and as such, basement membranes are likely to be a fundamental requirement for multi-cellular life ${ }^{1}$. In metazoa, basement membranes are integral regulators of tissue shape, size and health, and when disrupted they cause a range of diseases. Major and conserved basement membrane components include laminins, type IV collagen [G], nidogens [G] , and the heparan-sulphate proteoglycans [G] (HSPGs) agrin, perlecan and collagen XVIII [G] (Figure 1). These components establish a cell-adhesive sheet that maintains normal physiology by regulating many facets of cell biology, including cell polarity, proliferation, apoptosis, survival, migration, differentiation and signalling. Thus, basement membranes have a vital role in general maintenance of tissue homeostasis. In addition, they function as selective barriers between adjacent compartments in various tissues, including in lung alveoli, skin epidermis, gut epithelium, the placenta and the blood-brain barrier. One of the most studied basement membranes is in the kidney glomerulus. The glomerular basement membrane (GBM) separates podocytes from endothelial cells and contributes to the size and charge-selectivity of the glomerular filtration barrier, which allows water and small molecules to pass into the urinary space, but retains macromolecules and cells within the circulation. Indeed, the presence of haematuria or proteinuria is indicative of glomerular barrier dysfunction. Additional matrix compartments coexist in the glomerulus, namely the basement membrane of the Bowman's capsule, which is produced by the parietal epithelial cells and encapsulates the glomerular tuft; and the mesangial matrix, which is a loose interstitial matrix produced by mesangial cells that connect capillary loops in the glomerulus (Figure 2a). Although the mesangial matrix contains major basement membrane components it does not exist as a sheet-like structure.

The glomerular filtration barrier and the contribution of the GBM to barrier function was first investigated using ultrastructural and biochemical analyses ${ }^{2,3}$. These studies determined the three-layered structure of the barrier and identified major components but could not provide insights into the global composition of the GBM. More recently, unbiased approaches such as mass spectrometry (MS)-based proteomics have shown that the composition of basement membranes is tissue specific and revealed that the 
GBM is distinct from other basement membranes. For example, we now know that specific GBM components - including the a3a4a5 network of collagen IV, which predominates in the mature GBM and differs from the $\alpha 1 \alpha 1 \alpha 2$ network found in most basement membranes - are crucial for glomerular barrier function. Similarly, minor components of the GBM such as nephronectin and fibulin 1 have restricted tissue localization and likely provide important glomerular-specific functions. Unravelling the underlying reasons for these differences will improve our understanding of the diversity of basement membrane functions and potentially lead to new targeted therapeutic approaches. In this Review, we describe methods that have enabled investigation of GBM composition and structure and note how these studies have provided insights into GBM composition and assembly, its developmental transitions, and its role in glomerular filtration. We also describe GBM-associated diseases and discuss current and potential future treatments for these disorders.

\section{[H1] GBM composition and assembly}

\section{[H2] Hierarchical assembly}

During development, basement membrane assembly proceeds in a hierarchical fashion. In vitro analyses in embryoid bodies demonstrate that basement membrane assembly initiates with secretion of laminin [G] heterotrimers by endodermal cells into the extracellular space ${ }^{4}$. Polymerisation of this laminin generates an immature basement membrane, which consists of a lattice-like sheet that binds to specific receptors on the surface of cells including integrins, discoid domain receptors (DDRs), a-dystroglycan and syndecans ${ }^{5}$. Maturation of the basement membrane follows with the deposition and polymerisation of collagen IV, which establishes a reticular network on the laminin sheet. Formation of the GBM involves the fusion of two basement membranes that are independently generated by endothelial cells and podocytes during glomerulogenesis ${ }^{6}$. Thus, the mature GBM consists of two laminin sheets on either side of a thick, central collagenous network. Most basement membranes are 50 $100 \mathrm{~nm}$ in width. However, the GBM is much thicker, at $330-460 \mathrm{~nm}$ in humans ${ }^{7}$ and 50-300 nm in rodents ${ }^{8,9}$. The next stage in basement membrane assembly involves deposition of the HSPGs agrin and perlecan. In the mature GBM, agrin is the major $\mathrm{HSPG}^{10}$ expressed by podocytes and tethers laminins to cell surface receptors to augment adhesion. Agrin also connects laminin and collagen IV polymers via nidogen ${ }^{11}$. Glycosaminoglycan (GAG) side chains, which attach to agrin and the other major proteoglycans (perlecan and collagen XVIII), can bind water molecules, which probably contributes to the thickness of basement membranes in living animals ${ }^{12,13}$. 
Evidence for this hierarchical assembly of basement membranes comes from analysis of protein localization profiles during development in model organisms and the observation of embryonic lethality with the loss of basement membrane components. For example, an elegant genetic study used time-lapse imaging of endogenous GFPtagged basement membrane proteins in Drosophila to demonstrate the temporal hierarchy of basement membrane assembly ${ }^{14}$. This study showed that laminin $\alpha$ and $\beta$ subunits are expressed in the early Drosophila embryo, followed by expression of collagen IV and perlecan. This sequence of events fits the dynamic expression profiles of basement membrane orthologs in embryos of $C$. elegans ${ }^{15,16}$ and aligns with our understanding of basement membrane development from knockout studies in vertebrate models. In global laminin-deficient mice, basement membrane assembly is not initiated and embryos do not survive later than embryonic day (E) $5.5^{17}$. In contrast, global depletion of collagen IV in mice causes lethality later at E10.5-11.5 ${ }^{18}$. This lethality is not due to a lack of basement membrane assembly, but is rather caused by impaired stability as evidenced by the irregular appearance and ruptures of basement membranes throughout the embryo. Taken together, these findings support the hypothesis that the hierarchy of laminin sheet formation followed by assembly of collagen IV and then HSPG, as demonstrated in Drosophila, may be conserved in vertebrates.

\section{[H2] Basement membrane components}

[H3] Laminins

Laminins are the major non-collagenous components of basement membranes, and exist as heterotrimers composed of $\alpha, \beta$, and $\gamma$ chains. In the mature GBM, the major isoform is laminin $\alpha 5 \beta 2 \gamma 1$ (also known as termed laminin-521; Figure 1). Globular laminin (LG) domains exist at the C-terminus of the $\alpha$-chain of laminin, and bind to the $\alpha$-subunit of integrin receptors on the surface of cells. In podocytes, the major integrin receptor is $\alpha 3 \beta 1$, which interacts directly with laminin $\alpha 5$ via LG domains. As the laminin sheet assembles, the $\mathrm{N}$-terminal arms of the $\alpha, \beta$, and $\mathrm{y}$-chains interact to enable polymerisation. The laminin chain arms also contain variable numbers of laminin-type epidermal growth factor-like (LE) domains, which are important for the binding of laminin to nidogen ${ }^{19,20}$ and may act as a bridge to the collagen IV network, although this link has been disputed ${ }^{21}$. 


\section{[H3] Collagen}

Collagen IV is a major component of all basement membranes and is thought to provide tensile and compressive strength. It is encoded by six genes (COL4A1-6) in vertebrates, which form three triple helix trimers, $\alpha 1 \alpha 1 \alpha 2, \alpha 3 \alpha 4 \alpha 5$ and $\alpha 5 \alpha 5 \alpha 6$. Unlike laminins, the evolutionary appearance of collagen IV is restricted to metazoa ${ }^{1}$, suggesting that they were fundamental in the emergence of multicellularity. Collagen IV chains are large proteins that consist of a short, $N$-terminal $\sim 25$ amino acid (AA) 7S domain, a long $\sim 1,400$ AA collagenous domain, and a C-terminal $\sim 230$ AA noncollagenous domain. Interestingly, the central collagenous domain (which contains the Gly-X-Y sequences that permit triple helix formation) also contains about 20 short noncollagenous (or interruption) domains, which are thought to provide molecular flexibility ${ }^{22}$. Once trafficked out of the cell, the collagen IV chains assemble to form a polymer adjacent to the laminin sheet. The collagen IV network exists as a lattice with interprotomer binding via homophilic covalent crosslinking either between four neighbouring $\mathrm{N}$-terminal $7 \mathrm{~S}$ domains or between two adjoining non-collagenous domains at the $\mathrm{C}$-terminus. Of note the assembly of collagen IV polymers (specifically the oligomerisation of NC domains) only occurs outside cells as this process is dependent on chloride ions, which are more abundant in the extracellular space ${ }^{23}$. In the extracellular space, covalent crosslinking at the $7 \mathrm{~S}$ domains is mediated by lysyl oxidase-like 2 (LOXL2), creating a dodecamer that is a hallmark of collagen IV polymerisation ${ }^{24}$. Covalent crosslinking of opposing non-collagenous domains occurs via a sulfilimine bond ${ }^{25}$. Interestingly, formation of this bond is the only known requirement for ionic bromide in animals and requires the heme peroxidase, peroxidasin ${ }^{26}$.

\section{[H3] Heparan sulphate proteoglycans}

HSPGs are thought to provide basement membranes with negative charge and connect laminin and collagen IV networks, although their roles in GBM function seem to be dispensable as their depletion had no effect on glomerular development or function $^{27,28}$. The three major HSPGs expressed in the GBM during development are perlecan, agrin and collagen XVIII. Agrin is the primary HSPG synthesised by podocytes $^{28}$. The $\mathrm{N}$-terminal domain of agrin binds to laminin $\gamma 1$ to stabilise GAG side chains in a regular distribution within the GBM whereas its $C$-terminus binds to cellsurface receptors such as $\alpha$-dystroglycan and integrin $\alpha \mathrm{v} \beta 1$ to mediate cell-matrix adhesion and signalling ${ }^{29}$. Perlecan and collagen XVIII are abundant in most glomerular matrix compartments during glomerulogenesis before becoming restricted 
to the mesangial matrix and Bowman's capsule in the mature glomerulus ${ }^{28,30}$. Perlecan is found in the mature GBM, but only on the subendothelial side ${ }^{10}$ even in agrinknockout mice ${ }^{28}$. Collagen XVIII is a hybrid collagen-HSPG that is found on both sides of the mature GBM as a polarised molecule but is more abundant in the mesangial matrix and Bowman's capsule basement membrane ${ }^{31}$. Mice deficient in Col18a1 have impaired renal excretion, and show loss of glomerular stiffness, mild mesangial expansion and podocyte foot process effacement, but do not show evidence of ultrastructural GBM defects ${ }^{31}$, 32 . Of note, however, microindentation studies have shown the GBM in Col18a1-deficient mice is $30 \%$ softer than that of control mice ${ }^{29}$, suggesting a role for collagen XVIII in the mechanical properties of the GBM.

\section{[H3] Nidogens}

Nidogens are dumbbell-shaped sulphated monomeric glycoproteins that bind to collagen IV, HSPGs and laminin $\gamma 1^{33}$, 34. Nidogen-1 and nidogen-2 are both found in the $\mathrm{GBM}^{35}$, with nidogen-1 predominating in the adult $\mathrm{GBM}^{36,37}$. Nidogens are localised close to the collagen IV network ${ }^{38}$; they were initially thought to act as crosslinkers for laminins and collagen $\mathrm{IV}^{33}, 34$ and were therefore thought to be essential for basement membrane assembly. Although nidogens are crucial for basement membrane integrity in the lungs and heart, one study found that both nidogens were dispensable for kidney development and GBM assembly in mice, suggesting that nidogens have tissuespecific roles ${ }^{39}$. It is possible that other GBM proteins might compensate for the lack of nidogen in the kidney. Interestingly, in the skin, when collagen IV and laminin suprastructures were isolated from the dermal-epidermal basement membrane by immunomagnetic bead purification ${ }^{21}$, the core protein of perlecan was present in the laminin network, but the perlecan GAG side chains were present in the collagen IV network. Nidogens were found in both the laminin and collagen IV networks but did not form strong molecular bridges between the two polymers, suggesting that HSPGs, rather than nidogens, might act as the main bridge between laminins and collagen IV. However, as mentioned earlier deletion of perlecan or agrin did not affect glomerular architecture or function, suggesting that like nidogens, HSPGs are dispensable for GBM assembly ${ }^{27}$. However, support for a role of nidogens in GBM assembly and function comes from a study in mice lacking the specific binding site for nidogen-1 within laminin $\gamma 1$ (the $\gamma 1$ III4 LE module). These mice died immediately after birth with renal agenesis or non-functioning kidneys associated with defective glomeruli and focal GBM disruption ${ }^{40}$. Hence, the role of nidogens in GBM assembly is controversial 
and requires further investigation. To date, no genetic variants in nidogen have been associated with kidney phenotypes in humans.

\section{[H1] Studying GBM composition and structure}

\section{[H2] New insights into GBM composition}

Greater understanding of the composition of the GBM is important to elucidate mechanisms of GBM assembly and function in development, ageing and disease. Over the past 10 years tissue proteomics and genome-based bioinformatic studies have been used to generate species-specific catalogues of extracellular matrix and matrix-associated proteins within various healthy and diseased tissues. Collectively these studies have helped to define the matrisome; the catalogue of $>1,000$ mammalian genes that encode matrix and matrix-associated proteins.. ${ }^{41,42}$. A strategy that involved enrichment of the matrix from isolated glomeruli from human nephrectomy kidneys combined with unbiased MS-based proteomics ${ }^{37}$, 43 identified 144 structural and regulatory human matrix proteins, including 25 proteins known to be associated with the GBM (Figure 3). As it is not possible to isolate the GBM from other matrix compartments in the glomerulus, these proteins include those within the mesangial matrix and the Bowman's capsule basement membrane. Further investigations are therefore required to determine the abundance and role of specific proteins in the GBM.

An example of a protein detected in more than one compartment of the glomerular ECM is fibulin-1, which is a secreted matrix glycoprotein found in basement membranes, microfibrils and elastic fibres. Fibulin-1 can bind to other basement membrane proteins such as nidogen-1, and laminin $\alpha$ and $\gamma$ chains; these interactions are important for the structural integrity of these supramolecular matrix scaffolds within tissues $^{44}$. Fibulin-1 is found in both the mesangial matrix and the GBM, and is required for normal development of glomerular capillaries ${ }^{44,45}$. Mice lacking fibulin-1 develop distended capillary loops in $10-40 \%$ of glomeruli, and capillary wall malformations but do not show evidence of discrete GBM abnormalities ${ }^{45}$. In $C$. elegans, fibulin assembly is dependent on interaction with hemicentin via epidermal growth factor (EGF)-like domains at specific sites at which two basement membranes attach in the worm body ${ }^{46}$, 47. Hemicentin is an evolutionary conserved matrix glycoprotein and a member of the immunoglobulin superfamily. It is structurally similar to fibulin-1 and has been implicated in cell-cell and cell-matrix adhesion, and as a mediator of mitotic cytokinesis ${ }^{48,49}$. In $C$. elegans hemicentin aggregates with integrins and intracellular plakins [G] to form a specialised linkage system named B-LINK, which mediates the 
adhesion of adjacent basement membranes at specific uterine attachment sites ${ }^{50}$. A 2020 study investigated whether a similar role in GBM assembly exists for hemicentins in mice; however, hemicentin-1 was not detected in the mature GBM ${ }^{51}$. Furthermore, global deletion of Hmcn1 or Hmcn2 had no apparent effect on glomerulogenesis in mice or glomerular function at 10 months of age. Although this study detected hemicentin-1 in the mesangial matrix of mature glomeruli, its role in glomerular function remains unclear.

Global proteomic analyses in healthy mice also demonstrated that glomerular matrix composition differs according to genetic background and sex, and correlates with levels of albuminuria ${ }^{43}$. For example, levels of netrin-4, a member of the laminin superfamily, were higher in glomerular matrix extracts from FVB mice than in C57BL/J mice, which are more resistant to kidney disease. A subsequent study ${ }^{52}$ demonstrated that full-length netrin-4 forms high-affinity equimolar complexes with laminin $\gamma 1$ through laminin N-terminal (LN) domain interactions that block laminin polymerization and basement membrane assembly. That study also found that netrin-4 disrupts preformed laminin-111 ternary node complexes in vitro in a non-enzymatic manner, and exerts anti-angiogenic and anti-tumorigenic roles in vivo through its laminin-binding activity. A specific role for netrin-4 in GBM integrity is yet to be determined, but these studies suggest a possible causal relationship between the higher levels of netrin-4 in FVB mice and higher levels of albuminuria. The global proteomic analysis of healthy mice also showed a $60 \%$ overlap between the mouse and human glomerular matrisome as well as the existence of a shared set of known basement membrane components including: collagen IV $\alpha 3 \alpha 4 \alpha 5$; laminin-521, nidogens 1 and 2, and the HSPGs agrin, perlecan and type XVIII collagen ${ }^{43}$, supporting the proposal that major GBM components are conserved across species (Figure 3).

Podocytes and glomerular endothelial cells are likely to contribute distinct GBM components during kidney development ${ }^{53-55}$. Use of proteomics and transmission electron microscopy to characterise the matrix composition and morphology of matrices derived from human cultured podocytes and/or glomerular endothelial cells, showed that only co-culture of these two cell types jointly deposited a matrix that is molecularly and structurally similar to the glomerular matrix in vivo ${ }^{56}$. This finding supports the concept that the development and organisation of the GBM is facilitated by crosstalk between podocytes and glomerular endothelial cells. 


\section{[H2] Methods for visualization}

\section{[H3] Electron microscopy of the GBM}

Advanced electron microscopy (EM) techniques have provided detailed insights into the spatial relationship between glomerular cells and the GBM. One form of block-face scanning electron microscopy (SEM) called serial block-face SEM [G] (SBF-SEM) has been used to study the complex ultrastructure of the glomerulus and analyse the spatial configuration of the GBM in health and across a spectrum of kidney diseases at nanometre scale ${ }^{9,57,58}$. For instance, use of SBF-SEM demonstrated invasion of podocyte foot processes into damaged areas of GBM in various mouse models of glomerular disease ${ }^{9}$. Low-vacuum SEM [G] (LV-SEM), has been used to show the presence of spikes on the GBM and subepithelial electron-dense deposits in biopsy samples from patients with membranous nephropathy ${ }^{59}$, irregular GBM thickening and a basket-weave appearance in samples from patients with Alport syndrome ${ }^{60}$, and GBM thinning and perforation in samples from patients with thin basement membrane nephropathy $(T B M N)^{60,61}$. Another emerging SEM technique called helium ion microscopy [G] (HIM) ${ }^{62}$ has been used to reveal striking alterations on the surface of glomeruli from $\mathrm{Col}_{4} 3^{-1-}$ mice, including the deposition of long microfilaments, cytoplasmic bleb-like projections, and increased numbers of prominent podocyte bridge-like processes ${ }^{63}$. The use of this technology to investigate basement membranes is an exciting prospect for the future.

\section{[H3] Super resolution light microscopy}

Super resolution light microscopy enables imaging of tissue structures smaller than the diffraction limit of the illumination source, with EM-level precision ${ }^{64}$. The best known examples of Super resolution light microscopy approaches are stimulated emission depletion [G] (STED) and stochastic optical reconstruction microscopy [G] $(\mathrm{STORM})^{65}$. A landmark study used STORM to map the topographical localisation of GBM components at nanoscale resolution in healthy human and mouse. Based on antibody staining, the researchers demonstrated that the GBM has a layered structure in which collagen IV $\alpha 3 \alpha 4 \alpha 5$ trimers and nidogens are centrally located, collagen IV $\alpha 1 \alpha 2 \alpha 1$ trimers are located close to endothelial cells, and laminin-521 and agrin have their $\mathrm{N}$-terminal domains facing the interior of the GBM and $\mathrm{C}$-terminal domains oriented toward the surface of endothelial cells and podocytes. They also provided insight into the disruption in GBM organization that occurs in Col4a3 ${ }^{--}$(Alport) mice in which collagen IV $\alpha 3 \alpha 4 \alpha 5$ trimers were no longer detected and instead $\alpha 1 \alpha 2 \alpha 1$ trimers are spread across the GBM ${ }^{38}$. A subsequent study correlated molecular and 
structural changes identified by STORM and SEM imaging, respectively, in the podocyte actin cytoskeleton and slit diaphragms in mouse models of glomerular disease. For example, use of this approach identified loss of agrin in the sub-epithelial region of the GBM in Lamb2/- mice that also showed podocyte injury and displacement of slit diaphragm proteins nephrin and podocin away from the GBM ${ }^{66}$. STORM was also used to study the GBM integration of recombinant human laminin-521 following systemic injection in $\mathrm{Lamb2}^{-/}$Rag $1^{1-1}$ mice. This study reported accumulation of the recombinant laminin along the endothelial side of the GBM in these mutant mice, demonstrating the utility of super resolution light microscopy for defining molecular localization within the $\mathrm{GBM}^{67}$.

\section{[H3] Tissue expansion}

Tissue expansion [G] has also been used to image glomerular structures including the GBM, slit diaphragms and podocyte foot processes at nanoscale-resolution but using conventional diffraction-limited light microscopy ${ }^{68}$. Several protocols exist for expanding tissue, but the basic principles involve fixation and permeabilisation of samples, after which they are further embedded within a polymer meshwork and denatured. The samples are then uniformly expanded up to an average of 4.5 times their original dimension enabling subsequent visualisation of structures that were previously unresolved at a resolution of $200-\mathrm{nm}{ }^{69,70}$. Labelling with fluorescent antibodies can be performed before or after the samples are expanded. A 2018 study $^{71}$ that performed volumetric analysis and $3 \mathrm{D}$ reconstruction of podocyte foot processes imaged using tissue expansion and STED microscopy obtained a resolution of $<20 \mathrm{~nm}$ within the glomerular filtration barrier and thus the GBM.

\section{[H3] Decellularization approaches}

Decellularisation through chemical fractionation can also be used to analyse matrix by 3D imaging. In 2019, ${ }^{72}$ researchers published a detailed protocol for the in situ decellularisation of tissues (ISDoT) and 3D imaging of the resulting matrix scaffold. With the aid of multi-photon confocal microscopy and labelling with multiple antibodies, ISDoT enabled the interrogation of matrix proteins within decellularised matrix scaffolds of 33 different murine tissues. Perfusion of mice with decellularisation reagents (either $0.5 \%$ sodium deoxycholate or $0.1 \%$ sodium dodecyl sulphate) enabled the matrix scaffold to be observed in its native $3 \mathrm{D}$ context at a submicron level of resolution, with minimal or no structural damage. The application of such innovative techniques to the kidney is expected to provide new insights into the complexities of the GBM. 


\section{[H3] Protein tagging and live imaging}

Developments in technologies that aid our ability to visualise the GBM has improved our understanding of GBM composition, organisation and architecture; however, the imaging approaches that have been applied so far have used fixed tissue and therefore lack details about the dynamics of the GBM. New approaches that involve labelling of endogenous basement membrane components and live imaging using model systems have provided great insights into basement membrane dynamics including hierarchical assembly ${ }^{14}$ and turnover. For instance, studies with mCherry-tagged collagen IV and live imaging have tracked the spatiotemporal incorporation and turnover of collagen IV in $C$. elegans ${ }^{73,74}$. Indeed, the utility of $C$. elegans has been further demonstrated by a study that used gene editing to tag 29 major basement membrane proteins and receptors with $\mathrm{mNeonGreen,} \mathrm{providing} \mathrm{new} \mathrm{insights} \mathrm{into} \mathrm{interaction} \mathrm{between}$ basement membrane proteins, their dynamics and change during tissue maturation. Future approaches that involve labelling of endogenous GBM components and live imaging in appropriate experimental systems could provide similar insights into GBM dynamics including turnover and repair in the context of health and disease.

\section{[H1] GBM transitions during development}

In metanephric kidney development, glomerular precursors are first observed within the distal cleft of the S-shaped body ${ }^{75}, 76$. Secretion of VEGF by these primitive glomerular cells stimulates haemangioblasts [G] , which are most likely derived from the neighbouring mesenchyme, ${ }^{77}$ to form blood vessels that invade the distal cleft. The proximity of the glomerular precursors to the endothelium invokes a panoply of changes that gives rise to podocytes ${ }^{78}$. Mature podocytes are highly arborized and a key transition in their maturation is the replacement of classical epithelial cell-to-cell tight junctions with podocyte slit diaphragms composed of proteins such as nephrin, $\mathrm{NEPH} 1$ and podocin ${ }^{79-83}$. These cell-cell adhesion molecules are first expressed the S-shaped body stage and function to establish and maintain the slit diaphragm. Glomerular maturation creates a closed renal corpuscle encapsulated by a single layer of parietal epithelial cells (Bowman's capsule), which surrounds the capillary tuft and central supportive mesangium.

The GBM is formed from the fusion of two basement membranes that separately underlie the podocyte layer and the endothelium ${ }^{6}$. The fusing of two adjacent basement membranes is not unique to the GBM, and occurs in the optic cup ${ }^{84}$ and in lung alveoli85. The initial fusion of the early endothelial and podocyte basement membranes may require linking molecules. Given its role in basement membrane 
fusion in the worm, hemicentin was hypothesised to be a possible linking molecule in the GBM, though (as described above) recent evidence suggests hemicentin is not required for GBM formation ${ }^{49}$. In addition, formation of new GBM also occurs in situ as the glomerulus matures. The GBM assembly that occurs after the invasion of haemangioblasts into the distal cleft is unique in that the composition of the matrix undergoes defined changes that are essential for both filtration and continued development. These compositional transitions have been most intensely studied in terms of the contribution of laminins and collagen IV (Figure 2b). Laminin protein nomenclature labels trimers by their subunits such that laminin- $\alpha 5 \beta 2 \gamma 1$ is encoded by LAMA5, LAMB2 and LAMC1 and written as laminin-521. The major laminin heterotrimers expressed by early glomerular precursors are laminin-111 and laminin$511^{86,87}$ and these are replaced by laminin-521 as capillaries begin to form within the distal cleft of the S-shaped body ${ }^{88}$. The importance of replacing early laminin heterotrimers with laminin-521 is physiologically relevant as humans with pathogenic variants in LAMB2 develop Pierson syndrome, which is characterised by kidney, neurological and ocular defects ${ }^{89}$. Lama5 $^{-/}$mice exhibit loss of GBM integrity, which results in cessation of glomerulogenesis at the stage at which the a1 chain of laminin is replaced by laminin a5 heterotrimers ${ }^{90}$. These findings demonstrate that transitions observed in laminin heterotrimer composition in the early glomerulus are required for later glomerular development. However, grafting of Lama5 $5^{-/-}$metanephroi into wild type hosts resulted in glomerular vascularisation of the transplanted metanephroi ${ }^{91}$, although the laminin $\alpha 1$ chain was still detected in the GBM (predominantly on the podocyte side) and the podocyte slit diaphragm did not form. The observation that the hybrid GBM contained laminin a5 but predominantly on the wild-type endothelial side ${ }^{91}$ suggests that laminin $\alpha 5$ signalling is necessary for podocyte differentiation and highlights an essential role for the GBM in glomerulogenesis.

Simultaneously to the replacement of laminin- 111 and laminin- 511 by laminin-521 in the GBM, a switch in the collagen IV network occurs ${ }^{92}$ (Figure 2b). The early endothelial and podocyte basement membranes are composed of a collagen $\alpha 1 \alpha 1 \alpha 2$ network, but upon fusion of the two basement membranes an $\alpha 3 \alpha 4 \alpha 5$ network is deposited $^{92,93}$. Whether $\alpha 1 \alpha 1 \alpha 2$ is still produced by podocytes remains unclear but immunolabelling ${ }^{53}$, super resolution microscopy ${ }^{38}$ and genetic analyses ${ }^{94}$ suggest that the $a 3 a 4 a 5$ heterotrimer is exclusively synthesised by podocytes. The reason for this switch in the composition of the collagen IV network produced by podocytes is poorly understood; however, a reduction or absence of the a3a4a5 network causes Alport syndrome, which is characterised by kidney, hearing and ocular phenotypes ${ }^{95,} 96$. 
Although the $\alpha 1 \alpha 1 \alpha 2$ collagen IV network persists in human Alport kidneys, GBM thickness is reduced at early stages of the disease ${ }^{97}$, indicating that compensatory $\alpha 1 \alpha 1 \alpha 2$ synthesis is insufficient to maintain GBM integrity. In addition, the a3a4a5 heterotrimer is thought to provide additional biomechanical strength relative to that of the $\alpha 1 \alpha 1 \alpha 2$ heterotrimer. This increased strength may be conferred by the greater number of cysteine residues available to form intermolecular disulphide bonds between the $\alpha 3 a 4 a 5$ chains $^{98}$. The increased number of intermolecular bonds might also confer greater resistance to endopeptidase-mediated proteolysis ${ }^{97}$. Moreover, unique interactions between the $\alpha 3 \alpha 4 \alpha 5$ heterotrimer and cell membrane receptors (such as collagen IV binding integrins ${ }^{99}$ or discoidin domain receptors ${ }^{100,}{ }^{101}$ ) or matrix proteins (such as other $\alpha 3 \alpha 4 \alpha 5$ heterotrimers via disulphide bonding, or nidogen, and HSPGs) might also be important in strengthening and maintaining GBM integrity in mature glomeruli, but evidence for this role is currently lacking.

Other GBM components may also undergo developmentally timed changes in their expression and/or composition, but their profiles have not been formally investigated for functional significance. For example, immunostaining for perlecan shows that it is highly expressed throughout the immature GBM but is not expressed in the mature GBM where agrin predominates ${ }^{26}$. Furthermore, a potentially relevant switch in glycosaminoglycan (GAG) chain composition occurs in the GBM during development ${ }^{102}$. In early stages of development chondroitin sulphate is the dominant GAG in the GBM, but the mature GBM is composed mainly of heparan sulphate GAG chains on proteoglycans ${ }^{102}$. This change may be functionally relevant in disease as the thickened GBM that is associated with diabetic kidney disease is also abnormally associated with high levels of chondroitin sulphate proteoglycans ${ }^{103}$.

\section{[H1] The GBM in glomerular filtration}

Glomeruli filter approximately 180 litres of blood per day into the tubules, where $99 \%$ of the primary filtrate is reabsorbed and the remaining 1-2 litres of waste is excreted. Despite this seemingly simple function, the mechanism of size-selective filtration and the contribution of the GBM to filtration are not fully understood. The complex arrangement of podocyte foot processes prompted early modelling studies to describe the podocyte slit diaphragm as the major determinant of size selectivity. This concept was supported by measurements showing that the average spacing between podocyte foot processes is comparable to the size of albumin ${ }^{104,105}$. However, more recent discoveries have questioned this concept. 
The endothelial glycocalyx may also contribute to size-selective filtration in the glomerulus. The glycocalyx is a gel-like layer of proteins and GAG chains (including heparan sulphate, chondroitin sulphate and hyaluronan). It is positioned within the fenestrae of the endothelium and anchors down to the underlying $\mathrm{GBM}^{109}$. Studies in rodents show that enzymatic breakdown of GAGs within the endothelial glycocalyx ${ }^{110}$ or displacement of the glycocalyx by hypertonic sodium chloride ${ }^{111}$, induces an increase in albumin filtration by up to 12 -fold. However, the role of the endothelial glycocalyx in glomerular filtration has been questioned by in vivo experiments that implicated the GBM as the major component mediating size-selective filtration.

The gel-like resemblance of the GBM ${ }^{112}$ led to the proposal that the GBM follows Ogston's 1958 gel permeation principle, which describes how permeation into gels is related to molecular size ${ }^{113}$. This principle suggests that the GBM determines the permeation of macromolecules through the filter in a size-dependent manner through diffusion, whereas water and ions would pass through the filter by flow generated from hydraulic pressure ${ }^{112}$. In this model the fenestrated endothelium and the podocyte slit diaphragms do not act by size-selectivity, but rather provide resistance to the capillary fluid flow. Support for this gel permeation-diffusion model came from a 2017 study that used transmission electron microscopy to follow the fate of variably sized gold nanoparticles following their injection into the superior mesenteric artery of mice ${ }^{114}$. Large nanoparticles (equivalent in size to IgG dimers) permeated into the subendothelial GBM but did not enter its central region, whereas smaller nanoparticles (equivalent in size to IgG monomers) could partially permeate into the central region. Nanoparticles equivalent in size to albumin, which is much smaller than $\lg$ monomers, could permeate across the GBM. The researchers also showed that proximal tubules could completely absorb albumin-sized nanoparticles following tail vein injection at low concentration. However, the injection of high concentrations of albumin nanoparticles saturated the reabsorption capacity of the proximal tubules resulting in albuminuria. Interestingly, the gold nanoparticles used in these experiments frequently aggregated upstream of the slit diaphragms at the approximate position of the podocyte glycocalyx. They did not accumulate at the endothelial glycocalyx or at the protein bridges that form the slit diaphragm, leading to the conclusion that the GBM (acting as a relatively dense gel) and the podocyte glycocalyx are the two major sites that determine size selectivity in the glomerulus ${ }^{114}$. However, the reason why the podocyte glycocalyx acts as a barrier but the endothelial glycocalyx does not is unclear and requires further experimental insight. 
The gel permeation-diffusion model of glomerular filtration is therefore supported by experimental evidence; however, it is interesting to note that the absence of podocyte nephrin precludes the formation of the slit-diaphragm and causes massive proteinuria ${ }^{115,116}$ but has no known effect on GBM composition. Rather than providing evidence for an essential role of the slit diaphragm in size-selectivity, this finding may imply a requirement for the slit diaphragm in compression and tension of the GBM to maintain its physiological thickness. Indeed, a gel compression model ${ }^{117}$, proposes that podocyte foot process effacement limits GBM compression leading to a subtle, but physiologically relevant, shift from radial compression to circumferential tension. This shift reduces compression of the GBM, opening its meshwork and increasing the average pore size, enabling passage of larger macromolecules. Genetic models of kidney disease, such as $\mathrm{Lamb2}^{-1-}$ or $\mathrm{Col}_{4} \mathrm{a}^{-/}$mice, show podocyte foot process effacement and GBM thickening, which is consistent with a requirement for interdigitating podocyte foot processes in maintenance of GBM compression. In support of this model, a new study combined morphometric analyses of glomerular filters prior to and after the onset of albuminuria in a mouse model induced to develop glomerular barrier dysfunction ${ }^{118}$. Together with mathematical modelling, the researchers demonstrated that compression of the GBM by the 'buttress force' of podocyte foot processes facilitates permselectivity, and that when this function is perturbed capillary dilatation results, the GBM becomes more porous, and there albuminuria ensues.

The GBM and cellular glycocalyx maintain a net negative charge across the filtration barrier owing to the negative charge of HSPGs and their GAG side chains. This net anionic charge led to the suggestion that negatively charged molecules (such as albumin) would be repelled by the GBM and glycocalyx and selectively retained within the capillaries. However, this theory has been contested by several studies showing that depletion of HSPGs in the glomerulus does not affect glomerular function. For instance, one study showed that deletion of both agrin and perlecan reduced the net negative charge of the GFB in mice but did not induce proteinuria ${ }^{27}$. Similarly, removal of heparan sulphate side chains by glomerular overexpression of heparanase reduced GAG-associated anionic sites five-fold but did not cause changes in glomerular ultrastructure or function ${ }^{119}$. In zebrafish, mutation of the ext2 gene, which encodes the exostosin glycosyltransferse 2 that polymerizes GAG side chains attached to HSPGs, led to fewer anionic sites in the GBM but also did not induce proteinuria ${ }^{120}$. Together, these findings suggest that the role of charge selectivity in the glomerular filter is likely to be minor. However, despite the evidence in support of this conclusion, anionic 
molecule tracers have more difficulty in passing the glomerular filter than do neutral or cationic tracers ${ }^{121}$, suggesting that we do not fully understand the role of charge selectivity in the glomerulus.

\section{[H1] Human disease with GBM involvement}

Overt morphological changes in the GBM are seen in many glomerular diseases (Figure 4) and are caused by a multitude of molecular and biophysical mechanisms. Notable changes in GBM composition, arrangement and function occur in genetic diseases as well as acquired diseases, including autoimmune diseases such as antiGBM disease and membranous nephropathy, and metabolic diseases such as diabetic kidney disease.

\section{[H2] The spectrum of Alport syndrome}

Alport syndrome represents a spectrum of phenotypes caused by variants in the COL4A3, COL4A4 and COL4A5 genes. Patients typically present with early persistent haematuria followed by progressive proteinuria, and declining kidney function, often accompanied by hearing loss and ocular complications. These hallmarks are caused by progressive deterioration in the function of specific basement membranes and the progression of tissue fibrosis ${ }^{122}$. Early Alport syndrome is characterized by a thin GBM. However, as the disease progresses the GBM becomes segmentally thickened, split and lamellated giving rise to the characteristic basket-weave appearance ${ }^{9,}$ 122, 123. Podocyte foot process effacement and invasion into the GBM have also been observed $^{9,123,124}$. The COL4A3, COL4A4 and COL4A5 variants lead to inadequate incorporation of the collagen IV $\alpha 3 \alpha 4 \alpha 5$ network into the GBM during glomerulogenesis, which leads to retention of the collagen IV $\alpha 1 \alpha 1 \alpha 2$ network. Since the $\alpha 1 \alpha 1 \alpha 2$ trimers have about half the number of cysteine residues (and hence fewer disulfide bonds) than the $\alpha 3 \alpha 4 \alpha 5$ trimers $^{125}$, the abnormal GBM might have reduced mechanical resistance to the hydrostatic pressures that drive glomerular filtration. It has been proposed that the mechanical load imposed by filtration and the reduced compression imposed by effaced podocyte foot processes might therefore contribute to a progressive thickening of the GBM and thereby induce the onset of hematuria and proteinuria $^{117}$. The persistent $\alpha 1 \alpha 1 \alpha 2$ network in Alport syndrome is also thought to be more susceptible to proteolysis by matrix-degrading enzymes ${ }^{96,126,127}$, and can induce abnormal outside-in signaling via collagen IV binding receptors such as integrins $\alpha 1$ and $\alpha 2$, and discoidin domain receptor type-1 (DDR1). Deletion or inhibition of these receptors in $\mathrm{Col}_{4} \mathrm{a3}^{-1-}$ mice reduces podocyte injury and GBM damage and therefore 
delays progression in this model of Alport syndrome ${ }^{128-130}$. Accumulation of ectopic laminin $\alpha 1$ in thickened segments of the GBM has also been described in Col4a3 ${ }^{-1-}$ mice, associated with progressive loss of permeability to ferritin ${ }^{131}$, and might contribute to reduced podocyte adhesion ${ }^{132}$.

COL4A5 is located on the X chromosome and therefore males with X-linked AS (XLAS) are more severely affected than females. Females with XLAS have a single abnormal COL4A5 gene and an attenuated kidney phenotype with thin GBMs and some deposition of $\alpha 5$ subunits in the $\mathrm{GBM}^{133}$. Homozygous or compound heterozygous variants in COL4A3 or COL4A4 cause autosomal recessive Alport syndrome in both male and female. However, similar to XLAS in females, heterozygous variants in COL4A3 or COL4A4 cause thin basement membrane nephropathy (TBMN). GBM thickness in these individuals uniformly increases from a maximum of $180 \mathrm{~nm}$ in children (2-11 years-old) up to $200-264 \mathrm{~nm}$ in adults in at least $50 \%$ of the GBM in most glomeruli, which is still significantly thinner than that of normal adults (about $320 \mathrm{~nm}$ in females and $370 \mathrm{~nm}$ in males) ${ }^{134},{ }^{135}$. Typically, TBMN manifests as persistent microscopic haematuria in childhood; however, it is no longer considered benign as it is associated with progression to proteinuria and impaired kidney function in adults, with up to a $30 \%$ lifetime risk of kidney failure. Unlike XLAS in males and autosomal recessive AS, the $\alpha 3 \alpha 4 \alpha 5$ network is present in the mature GBM in TBMN but the GBM is diffusely thinned and use of LV-SEM has demonstrated the existence of irregular holes along the GBM surface ${ }^{61}$. The GBM thinning is likely explained by a gene dosage effect whereby podocytes that carry one abnormal copy of COL4A3, COL4A4 or COL4A5 produce fewer $\alpha 3 \alpha 4 \alpha 5$ trimers than podocytes that carry normal copies of both genes ${ }^{134}$. Individuals with heterozygous variants in any of these genes and poor prognostic indicators (e.g., hypertension, proteinuria) should receive lifelong renal surveillance ${ }^{122}$.

\section{[H2] Pierson syndrome}

Pierson syndrome is a rare genetic disorder caused by variants in $\angle A M B 2$, which result in a complete or partial lack of laminin-521 heterotrimers in the GBM, eye and muscle tissue. The clinical features of Pierson syndrome include nephrotic syndrome with progression to kidney failure within the first year of life, neurological defects, and microcoria [G] ${ }^{136}$. Studies of Lamb2-- mice have contributed enormously to the pathophysiological underpinnings of GBM dysfunction in Pierson syndrome. These mice develop normally in utero and assemble a structurally intact GBM in which the 
lack of laminin-521 is compensated for by ectopic laminins-111, -211, -332 and more commonly -511 but not at sufficient levels to secure long-term maintenance of glomerular permselectivity. Newborn Lamb2 ${ }^{-1}$ mice develop massive proteinuria shortly after birth that progress to nephrotic syndrome three weeks after birth ${ }^{137-139}$. Severe podocyte foot process effacement is detected at two weeks after birth, and although the GBM is ultrastructurally intact in EM preparations, it displays disorganization of anionic sites and increased permeability to ferritin ${ }^{139}$. Notably, since proteinuria appears before signs of morphological change in the GBM or podocytes, it has been suggested that proteinuria in $\angle A M B 2$ deficiency is primarily caused by a permselectivity defect in the $\mathrm{GBM}^{139}$. This concept is supported by the observation that the degree of ferritin permeability in the GBM in $\mathrm{Lamb2}^{-1}$ mice is not dependent on normal podocyte morphology. Interestingly, an ultrastructural analysis of kidney biopsy samples from patients with a spectrum of glomerular diseases that cause nephrotic syndrome revealed the presence of tunnels and cavities throughout the nephrotic GBM apparently large enough to allow unrestricted protein leakage even in the absence of GBM thickening ${ }^{140}$. Since laminins are essential for basement membrane assembly, it has been proposed that the lack of laminin-521 could permit similar fluid flow through the GBM as a result of altered structural organization due to the defective laminin network, thereby causing massive early proteinuria ${ }^{138}$ 139, 141. An alternative explanation is that the signalling axis from laminin-521 in the GBM via $\alpha 3 \beta 1$ integrins to the podocyte cytoskeleton is critical for the maintenance of podocyte function and glomerular permselectivity. Indeed distinct basement membrane ligands have been shown to influence both cell shape and the assembly of adhesion complexes in podocytes ${ }^{130}$, indicating that the matrix ligand matters. Furthermore, forced podocytespecific overexpression of Lamb1 in Lamb2 $2^{--}$mice restored glomerular function, attenuated GBM damage and podocyte injury in a dose-dependent manner ${ }^{141}$. This finding suggests outside-in signalling is also key for intact glomerular function and that the density of laminin polymers within the GBM may be an important factor.

\section{[H2] Autoimmune disease}

[H3] Anti-GBM disease

In addition to genetic disorders, autoimmune disease can also cause abnormalities in basement membranes. Anti-GBM disease, which is a feature of Goodpasture syndrome, is a rare, immune-mediated condition caused by autoantibodies against conformational and cryptic epitopes in collagen IV. The dominant target antigen is in the NC-1 domain of the $\alpha 3$ chain $^{142}$, and the disease is characterized by the linear 
deposition of anti- $\alpha 3$ (IV)NC1 lgG (and often complement component C3) along the full length of the GBM with an absence of electron-dense deposits ${ }^{143-145}$. Patients with antiGBM disease (mostly adults) develop glomerular injury characterised by a crescentic or focal necrotising glomerulonephritis and a rapid decline in kidney function.

[H3] Membranous nephropathy

GBM defects also occur in autoimmune diseases that target podocytes antigens. Membranous nephropathy is the most common cause of immune-mediated nephrotic syndrome in adults and its idiopathic form is associated with autoantibodies against surface antigens expressed by podocytes, predominantly the phospholipase A2 receptor ( $70 \%$ of cases) and thrombospondin domain $7 A\left(2-5 \%\right.$ of cases) ${ }^{146}$. The GBM of patients with membranous nephropathy is profoundly thickened with subepithelial electron-dense deposits and characteristic matrix spikes between the deposits. The dense deposits contain lgG (mostly lgG4) and complement components (C3, C4 and C5b-9), which are trapped within the subepithelial surface of the GBM, although the mechanism for this trapping remains unclear. Spontaneous remission occurs in 30$40 \%$ of cases, but $40 \%$ of patients with persistent proteinuria progress to kidney failure within 10 years ${ }^{146-148}$. Of note membranous nephropathy is associated with loss of GBM heparan sulphate GAGs in patients ${ }^{149}$ and in a rat model of membranous nephropathy ${ }^{150}$. Given that heparan sulphate GAGs regulate complement activation via interactions with complement regulatory proteins, decreased heparan sulphate content in the GBM might be critical for glomerular damage induced by in situ activation of the complement system ${ }^{151}$.

[H2] Diabetic kidney disease

GBM thickening is an early morphological feature of diabetic kidney disease (DKD) followed by mesangial matrix expansion and glomerulosclerosis, accompanied by the development of proteinuria and a progressive decline in kidney function ${ }^{152-155}$. A 2019 study identified GBM thickness as a predictor of renal survival in patients with DKD ${ }^{156}$. Ultrastructural studies of the GBM in patients with advanced DKD have revealed dramatic changes including denudation and abnormal folding of the GBM, and the presence of shallow crater-like cavities and tunnels in fragmented segments of the GBM $^{153,157}$. GBM thickening is generally thought to result from matrix dysregulation, involving overproduction of matrix components and low rates of turnover in response to the activation of inflammatory and profibrotic pathways linked to high glucose levels. Diminished expression and activity of matrix-degrading enzymes and their natural inhibitors might also contribute to the accumulation of matrix in DKD ${ }^{155,}{ }^{158-160}$. 
Depletion of intracellular ATP in response to high glucose levels also decreases the de novo synthesis and post-translational processing of HSPGs and heparan sulfate GAGs, which become scarce in the thickened GBM $^{154,155,161}$. The severity of GBM damage in DKD is also associated with the formation of advanced glycation end products [G] (AGEs). For example, collagen IV is highly susceptible to damage by AGEs at multiple functional sites ${ }^{162,163}$, and AGEs can disrupt cell-matrix interactions and enhance the number of crosslinking bonds within basement membrane proteins in a way that disturbs their assembly and turnover ${ }^{162-164}$.

DKD is known to have an underlying genetic component ${ }^{165}$. In the past few years, two variants in COL4A3 have been associated with disease progression in DKD. The first, a low-frequency single nucleotide polymorphism (rs34505188 causing a Arg408His substitution) was detected in African Americans with DKD and was predicted to be pathogenic owing to its localisation within a highly conserved region of the gene ${ }^{166}$. The second, a common single nucleotide polymorphism (rs55703767 causing a Asp326Tyr substitution) was detected in Europeans with type 1 diabetes mellitus. This variant might provide protection against albuminuria and GBM thickening as the amino acid substitution could destabilise the $\alpha 3 \alpha 4 \alpha 5$ trimer, resulting in a more flexible network that resists the forces induced by hyperfiltration and is more accessible to proteolytic degradation, enabling faster turnover ${ }^{167,168}$. Further functional studies of these COL4A3 variants, in addition to variants in other basement membrane genes could improve understanding of basement membrane biology and the roles of GBM components in glomerular disease.

\section{[H1] Therapy for GBM-associated disease}

No curative therapies currently exist for GBM-associated disease; however, a number of treatments can prolong kidney survival (Table 1). Renin-angiotensin-aldosterone system (RAAS) inhibitors are the current mainstay of therapy for Alport syndrome, and aim to reduce intraglomerular pressure and thereby mechanical load on glomerular capillaries, This therapy reduces proteinuria and delays kidney failure by a decade or more ${ }^{122}$. New therapy prospects that aim to delay disease progression have also emerged from preclinical studies (Table 1).

One line of investigation is the assessment of approaches that target fibrosis. Some of these agents, such as TGF- $\beta$ inhibitors and collagen IV receptor blockers, are still in the preclinical phase of investigation, whereas others, including a microRNA-21 
inhibitor (ClinicalTrials.gov Identifier: NCT02855268) ${ }^{169}$, are currently in clinical trials. Future strategies to correct basement membrane defects could also address protein trafficking (with aid of chemical chaperone) in Alport syndrome ${ }^{170}$ or deleterious posttranslational modifications such formation of AGEs in diabetes ${ }^{155}$. In support of this, studies have shown that attenuation of AGE signalling through the receptor, RAGE, ameliorated oxidative stress, GBM thickening, glomerulosclerosis and albuminuria in rodent models of DKD ${ }^{155,171 .}$

Approaches to correct the defects in GBM composition to re-establish function and prevent disease progression are also an avenue of investigation. For example, transgenic expression of the human COL4A3-COL4A4 locus rescued the Alport syndrome phenotype of $\mathrm{Co} / 4 \mathrm{a}^{-/-}$mice ${ }^{172}$. Similarly, transgenic expression of podocytespecific Col4a3 after the onset of proteinuria in $\mathrm{Col}_{4 a 3^{--}}$mice partially rescued the collagen IV network and the ultrastructure of in the $\mathrm{GBM}^{173}$. These findings suggest that gene replacement therapies might be effective in Alport syndrome. Indeed the therapeutic potential of gene-targeting strategies is also being investigated for GBMassociated disease (Figure 5). A 2019 study reported the successful use of CRISPRCas9 gene editing in cultured urine-derived podocytes from patients with Alport syndrome. ${ }^{174}$ This approach achieved homologous correction in $59 \%$ of variant in COL4A5 (Gly624Asp) and 44\% of variants in COL4A3 (Gly856Glu) ${ }^{174}$. Exon skipping therapy [G] can also be induced by CRISPR-Cas9, or with splice-blocking antisense oligonucleotides. A study in a mouse model of Joubert syndrome [G] showed that systemic treatment with a splice site masking-antisense oligonucleotide partially rescued full-length transcript and protein levels of CEP290, and ameliorated cystic kidney disease ${ }^{175}$. Exon skipping has also been used to target truncating variants in exon 21 of $C O L 4 A 5$ in a mouse model of Alport syndrome ${ }^{176}$. The intervention resulted in successful collagen IV $\alpha 3 \alpha 4 \alpha 5$ trimer assembly and GBM localisation and extended kidney survival in mice. These findings provide optimism for human translation and the treatment of severe truncating variants in COL4A5.

Recombinant proteins can also be efficiently delivered to the GBM and this approach represents another potential therapeutic option for GBM-associated disease (Figure 5). Daily injection of pre-albuminuric Lamb2 $^{-/-}$mice with recombinant human laminin521 restored GBM permselectivity and delayed proteinuria ${ }^{67}$. The human laminin-521 stably accumulated on the endothelial side of the GBM but did not accumulate in the GBM on the podocyte side. Despite this encouraging observation these mice still developed nephrotic syndrome, perhaps as a consequence of absent laminin-521 
engagement with podocyte adhesion receptors. Vascular delivery of smaller GBMrepair proteins is another potential strategy. Linker proteins, which are small versions of full-length matrix proteins (for example, $\alpha \mathrm{LNNd}$ ) or chimeric engineered peptide sequences (for example, miniagrin), are also being used to rescue function and to identify mechanisms by which amino acid substitutions in laminin LN domains affect basement assembly (Figure 5). In vitro experiments demonstrated that the linker protein $\beta \mathrm{LNNd}$ can reestablish polymerisation of a non-polymerising laminin-111 that bears a mutation in its $\beta 1 \mathrm{LN}$ domain (LM $\beta 1$ Ser68Arg, which is homologous to LM $\beta 2$ Ser80Arg that leads to Pierson syndrome) and can assemble with collagen IV and nidogen ${ }^{177}$. These findings suggest that engineered linker proteins could also be an effective approach to repair GBM defects in conditions such as Pierson syndrome. The challenges for this approach include mapping the precise interactions of GBM components, identifying the location of key binding domains for targeting defective proteins, and the design and development of strategic and efficient delivery systems.

One goal of future therapy for GBM-associated disease would be to exploit endogenous mechanisms of GBM repair; however, the regulated maintenance, turnover and potential for repair of basement membranes is currently poorly understood. The GBM undergoes turnover, although the exact timescale of this remodeling is unclear. One of the very few studies of GBM turnover published in 1972 assessed two patients following excessive exposure to silver. In one patient, silver was identified in the GBM 12 weeks after exposure to silver; in the other case it was absent from the GBM 14 years following exposure ${ }^{178}$ and is therefore inconclusive with regard to the timescale of GBM turnover. Current approaches to label endogenous matrix proteins $^{74,179}$ will ultimately improve our understanding of GBM turnover and the potential for basement membrane repair.

\section{[H1] Conclusions}

The GBM represents a highly specialised macromolecular structure that is uniquely designed to facilitate filtration. Studies of GBM composition using emerging imaging and proteomics technologies continue to build a picture of the complexity of the GBM and provide insights into how composition and structure relate to GBM function. GBM defects are seen across a wide spectrum of glomerular diseases. As our understanding of GBM assembly, maintenance and repair improves so will our ability to conceive new therapeutic strategies to stabilise or even repair defective basement membranes. 


\section{References}

1. Fidler, A.L. et al. Collagen IV and basement membrane at the evolutionary dawn of metazoan tissues. Elife 6 (2017).

2. Farquhar, M.G. Editorial: The primary glomerular filtration barrier--basement membrane or epithelial slits? Kidney international 8, 197-211 (1975).

3. Timpl, R. Recent advances in the biochemistry of glomerular basement membrane. Kidney international 30, 293-298 (1986).

4. Li, S., Edgar, D., Fässler, R., Wadsworth, W. \& Yurchenco, P.D. The role of laminin in embryonic cell polarization and tissue organization. Developmental cell 4, 613-624 (2003).

5. Hohenester, E. \& Yurchenco, P.D. Laminins in basement membrane assembly. Cell adhesion \& migration 7, 56-63 (2013).

6. Abrahamson, D.R. Origin of the glomerular basement membrane visualized after in vivo labeling of laminin in newborn rat kidneys. The Journal of cell biology 100, 1988-2000 (1985).

7. Dische, F.E. Measurement of glomerular basement membrane thickness and its application to the diagnosis of thin-membrane nephropathy. Arch Pathol Lab Med 116, 43-49 (1992).

8. Neumann, K.H., Kellner, C., Kühn, K., Stolte, H. \& Schurek, H.J. Age-dependent thickening of glomerular basement membrane has no major effect on glomerular hydraulic conductivity. Nephrol Dial Transplant 19, 805-811 (2004).

9. Randles, M.J. et al. Three-dimensional electron microscopy reveals the evolution of glomerular barrier injury. Scientific reports 6, 35068 (2016).

10. Groffen, A.J. et al. Agrin is a major heparan sulfate proteoglycan in the human glomerular basement membrane. The journal of histochemistry and cytochemistry : official journal of the Histochemistry Society 46, 19-27 (1998).

11. Fox, J.W. et al. Recombinant nidogen consists of three globular domains and mediates binding of laminin to collagen type IV. The EMBO journal 10, 3137-3146 (1991).

12. Candiello, J., Cole, G.J. \& Halfter, W. Age-dependent changes in the structure, composition and biophysical properties of a human basement membrane. Matrix Biol 29, 402-410 (2010).

13. Balasubramani, M. et al. Molecular interactions in the retinal basement membrane system: a proteomic approach. Matrix Biol 29, 471-483 (2010).

14. Matsubayashi, Y. et al. A Moving Source of Matrix Components Is Essential for De Novo Basement Membrane Formation. Curr Biol 27, 3526-3534.e3524 (2017).

15. Graham, P.L. et al. Type IV collagen is detectable in most, but not all, basement membranes of Caenorhabditis elegans and assembles on tissues that do not express it. The Journal of cell biology 137, 1171-1183 (1997).

16. Huang, C.C. et al. Laminin alpha subunits and their role in C. elegans development. Development (Cambridge, England) 130, 3343-3358 (2003).

17. Smyth, N. et al. Absence of basement membranes after targeting the LAMC1 gene results in embryonic lethality due to failure of endoderm differentiation. The Journal of cell biology 144, 151-160 (1999).

18. Poschl, E. et al. Collagen IV is essential for basement membrane stability but dispensable for initiation of its assembly during early development. Development 131, 1619-1628 (2004).

19. Stetefeld, J., Mayer, U., Timpl, R. \& Huber, R. Crystal structure of three consecutive laminintype epidermal growth factor-like (LE) modules of laminin gamma1 chain harboring the nidogen binding site. J Mol Biol 257, 644-657 (1996).

20. Baumgartner, R. et al. Structure of the nidogen binding LE module of the laminin gamma1 chain in solution. J Mol Biol 257, 658-668 (1996).

21. Behrens, D.T. et al. The epidermal basement membrane is a composite of separate laminin- or collagen IV-containing networks connected by aggregated perlecan, but not by nidogens. The Journal of biological chemistry 287, 18700-18709 (2012).

22. Xu, T., Zhou, C.Z., Xiao, J. \& Liu, J. Unique Conformation in a Natural Interruption Sequence of Type XIX Collagen Revealed by Its High-Resolution Crystal Structure. Biochemistry 57, 1087-1095 (2018).

23. Cummings, C.F. et al. Extracellular chloride signals collagen IV network assembly during basement membrane formation. The Journal of cell biology 213, 479-494 (2016).

24. Anazco, C. et al. Lysyl Oxidase-like-2 Cross-links Collagen IV of Glomerular Basement Membrane. The Journal of biological chemistry 291, 25999-26012 (2016). 
25. McCall, A.S. et al. Bromine is an essential trace element for assembly of collagen IV scaffolds in tissue development and architecture. Cell 157, 1380-1392 (2014).

26. Bhave, G. et al. Peroxidasin forms sulfilimine chemical bonds using hypohalous acids in tissue genesis. Nature chemical biology 8, 784-790 (2012).

27. Goldberg, S., Harvey, S.J., Cunningham, J., Tryggvason, K. \& Miner, J.H. Glomerular filtration is normal in the absence of both agrin and perlecan-heparan sulfate from the glomerular basement membrane. Nephrology, dialysis, transplantation : official publication of the European Dialysis and Transplant Association - European Renal Association 24, 2044-2051 (2009).

28. Harvey, S.J. et al. Disruption of glomerular basement membrane charge through podocytespecific mutation of agrin does not alter glomerular permselectivity. Am J Pathol 171, 139-152 (2007).

29. Groffen, A.J., Veerkamp, J.H., Monnens, L.A. \& van den Heuvel, L.P. Recent insights into the structure and functions of heparan sulfate proteoglycans in the human glomerular basement membrane. Nephrol Dial Transplant 14, 2119-2129 (1999).

30. Saarela, J., Rehn, M., Oikarinen, A., Autio-Harmainen, H. \& Pihlajaniemi, T. The short and long forms of type XVIII collagen show clear tissue specificities in their expression and location in basement membrane zones in humans. Am J Pathol 153, 611-626 (1998).

31. Kinnunen, A.I. et al. Lack of collagen XVIII long isoforms affects kidney podocytes, whereas the short form is needed in the proximal tubular basement membrane. J Biol Chem 286, 77557764 (2011).

32. Utriainen, A. et al. Structurally altered basement membranes and hydrocephalus in a type XVIII collagen deficient mouse line. Hum Mol Genet 13, 2089-2099 (2004).

33. Aumailley, M., Wiedemann, H., Mann, K. \& Timpl, R. Binding of nidogen and the lamininnidogen complex to basement membrane collagen type IV. Eur J Biochem 184, 241-248 (1989).

34. Aumailley, M. et al. Nidogen mediates the formation of ternary complexes of basement membrane components. Kidney Int 43, 7-12 (1993).

35. Miner, J.H. The glomerular basement membrane. Exp Cell Res 318, 973-978 (2012).

36. Miosge, N. et al. Ultrastructural colocalization of nidogen-1 and nidogen-2 with laminin-1 in murine kidney basement membranes. Histochem Cell Biol 113, 115-124 (2000).

37. Lennon, R. et al. Global analysis reveals the complexity of the human glomerular extracellular matrix. J Am Soc Nephrol 25, 939-951 (2014).

38. Suleiman, H. et al. Nanoscale protein architecture of the kidney glomerular basement membrane. Elife 2, e01149 (2013).

39. Bader, B.L. et al. Compound genetic ablation of nidogen 1 and 2 causes basement membrane defects and perinatal lethality in mice. Molecular and cellular biology 25, 6846-6856 (2005).

40. Willem, M. et al. Specific ablation of the nidogen-binding site in the laminin gamma1 chain interferes with kidney and lung development. Development 129, 2711-2722 (2002).

41. Hynes, R.O. \& Naba, A. Overview of the matrisome--an inventory of extracellular matrix constituents and functions. Cold Spring Harb Perspect Biol 4, a004903 (2012).

42. Naba, A. et al. The matrisome: in silico definition and in vivo characterization by proteomics of normal and tumor extracellular matrices. Mol Cell Proteomics 11, M111.014647 (2012).

43. Randles, M.J. et al. Genetic Background is a Key Determinant of Glomerular Extracellular Matrix Composition and Organization. Journal of the American Society of Nephrology : JASN 26, 3021-3034 (2015).

44. de Vega, S., Iwamoto, T. \& Yamada, Y. Fibulins: multiple roles in matrix structures and tissue functions. Cellular and molecular life sciences : CMLS 66, 1890-1902 (2009).

45. Kostka, G. et al. Perinatal lethality and endothelial cell abnormalities in several vessel compartments of fibulin-1-deficient mice. Mol Cell Biol 21, 7025-7034 (2001).

46. Muriel, J.M., Dong, C., Hutter, H. \& Vogel, B.E. Fibulin-1C and Fibulin-1D splice variants have distinct functions and assemble in a hemicentin-dependent manner. Development 132, 4223-4234 (2005).

47. Muriel, J.M., Dong, C. \& Vogel, B.E. Distinct regions within fibulin-1D modulate interactions with hemicentin. Exp Cell Res 318, 2543-2547 (2012).

48. Xu, X. et al. Specific structure and unique function define the hemicentin. Cell Biosci 3, 27 (2013).

49. Morrissey, M.A. \& Sherwood, D.R. An active role for basement membrane assembly and modification in tissue sculpting. J Cell Sci 128, 1661-1668 (2015). 
50. Morrissey, M.A. et al. B-LINK: a hemicentin, plakin, and integrin-dependent adhesion system that links tissues by connecting adjacent basement membranes. Developmental cell 31, 319-331 (2014).

51. Lin, M.H. et al. Mammalian hemicentin 1 is assembled into tracks in the extracellular matrix of multiple tissues. Dev Dyn (2020).

52. Reuten, R. et al. Structural decoding of netrin-4 reveals a regulatory function towards mature basement membranes. Nat Commun 7, 13515 (2016).

53. Abrahamson, D.R., Hudson, B.G., Stroganova, L., Borza, D.B. \& St John, P.L. Cellular origins of type IV collagen networks in developing glomeruli. Journal of the American Society of Nephrology : JASN 20, 1471-1479 (2009).

54. Abrahamson, D.R., St John, P.L., Stroganova, L., Zelenchuk, A. \& Steenhard, B.M. Laminin and type IV collagen isoform substitutions occur in temporally and spatially distinct patterns in developing kidney glomerular basement membranes. J Histochem Cytochem 61, 706-718 (2013).

55. St John, P.L. \& Abrahamson, D.R. Glomerular endothelial cells and podocytes jointly synthesize laminin-1 and -11 chains. Kidney Int 60, 1037-1046 (2001).

56. Byron, A. et al. Glomerular cell cross-talk influences composition and assembly of extracellular matrix. Journal of the American Society of Nephrology : JASN 25, 953-966 (2014).

57. Arkill, K.P. et al. Resolution of the three dimensional structure of components of the glomerular filtration barrier. BMC Nephrol 15, 24 (2014).

58. Takaki, T., Ohno, N., Saitoh, S., Nagai, M. \& Joh, K. Podocyte penetration of the glomerular basement membrane to contact on the mesangial cell at the lesion of mesangial interposition in lupus nephritis: a three-dimensional analysis by serial block-face scanning electron microscopy. Clin Exp Nephrol 23, 773-781 (2019).

59. Miyazaki, H. et al. Application of low-vacuum scanning electron microscopy for renal biopsy specimens. Pathol Res Pract 208, 503-509 (2012).

60. Okada, S. et al. Morphological diagnosis of Alport syndrome and thin basement membrane nephropathy by low vacuum scanning electron microscopy. Biomed Res 35, 345-350 (2014).

61. Kajimoto, Y. et al. Pathologic glomerular characteristics and glomerular basement membrane alterations in biopsy-proven thin basement membrane nephropathy. Clin Exp Nephrol 23, 638649 (2019).

62. Joens, M.S. et al. Helium Ion Microscopy (HIM) for the imaging of biological samples at subnanometer resolution. Sci Rep 3, 3514 (2013).

63. Tsuji, K. et al. Ultrastructural Characterization of the Glomerulopathy in Alport Mice by Helium Ion Scanning Microscopy (HIM). Sci Rep 7, 11696 (2017).

64. Pullman, J.M. New Views of the Glomerulus: Advanced Microscopy for Advanced Diagnosis. Front Med (Lausanne) 6, 37 (2019).

65. Tam, J. \& Merino, D. Stochastic optical reconstruction microscopy (STORM) in comparison with stimulated emission depletion (STED) and other imaging methods. J Neurochem 135, 643658 (2015).

66. Suleiman, H.Y. et al. Injury-induced actin cytoskeleton reorganization in podocytes revealed by super-resolution microscopy. JCI Insight 2 (2017).

67. Lin, M.H. et al. Laminin-521 Protein Therapy for Glomerular Basement Membrane and Podocyte Abnormalities in a Model of Pierson Syndrome. J Am Soc Nephrol 29, 1426-1436 (2018).

68. Chozinski, T.J. et al. Volumetric, Nanoscale Optical Imaging of Mouse and Human Kidney via Expansion Microscopy. Sci Rep 8, 10396 (2018).

69. Angelotti, M.L., Antonelli, G., Conte, C. \& Romagnani, P. Imaging the kidney: from light to super-resolution microscopy. Nephrol Dial Transplant (2019).

70. Wassie, A.T., Zhao, Y. \& Boyden, E.S. Expansion microscopy: principles and uses in biological research. Nat Methods 16, 33-41 (2019).

71. Unnersjö-Jess, D. et al. Confocal super-resolution imaging of the glomerular filtration barrier enabled by tissue expansion. Kidney Int 93, 1008-1013 (2018).

72. Mayorca-Guiliani, A.E. et al. Decellularization and antibody staining of mouse tissues to map native extracellular matrix structures in 3D. Nat Protoc 14, 3395-3425 (2019).

73. Jayadev, R. et al. $\alpha$-Integrins dictate distinct modes of type IV collagen recruitment to basement membranes. J Cell Biol 218, 3098-3116 (2019).

74. Morrissey, M.A. et al. SPARC Promotes Cell Invasion In Vivo by Decreasing Type IV Collagen Levels in the Basement Membrane. PLoS Genet 12, e1005905 (2016). 
75. Tufro, A., Norwood, V.F., Carey, R.M. \& Gomez, R.A. Vascular endothelial growth factor induces nephrogenesis and vasculogenesis. Journal of the American Society of Nephrology : JASN 10, 2125-2134 (1999).

76. Kitamoto, Y., Tokunaga, H. \& Tomita, K. Vascular endothelial growth factor is an essential molecule for mouse kidney development: glomerulogenesis and nephrogenesis. The Journal of clinical investigation 99, 2351-2357 (1997).

77. Ballermann, B.J. Glomerular endothelial cell differentiation. Kidney international 67, 16681671 (2005).

78. Majumdar, A. \& Drummond, I.A. Podocyte differentiation in the absence of endothelial cells as revealed in the zebrafish avascular mutant, cloche. Developmental genetics 24, 220-229 (1999).

79. Holzman, L.B. et al. Nephrin localizes to the slit pore of the glomerular epithelial cell. Kidney international 56, 1481-1491 (1999).

80. Ruotsalainen, V. et al. Role of nephrin in cell junction formation in human nephrogenesis. The American journal of pathology 157, 1905-1916 (2000).

81. Liu, G. et al. Neph1 and nephrin interaction in the slit diaphragm is an important determinant of glomerular permeability. The Journal of clinical investigation 112, 209-221 (2003).

82. Roselli, S. et al. Podocin Localizes in the Kidney to the Slit Diaphragm Area. The American journal of pathology 160, 131-139 (2002).

83. Reiser, J., Kriz, W., Kretzler, M. \& Mundel, P. The glomerular slit diaphragm is a modified adherens junction. Journal of the American Society of Nephrology : JASN 11, 1-8 (2000).

84. Tsuji, N., Kita, K., Ozaki, K., Narama, I. \& Matsuura, T. Organogenesis of mild ocular coloboma in FLS mice: failure of basement membrane disintegration at optic fissure margins. Experimental eye research 94, 174-178 (2012).

85. Vaccaro, C.A. \& Brody, J.S. Structural features of alveolar wall basement membrane in the adult rat lung. The Journal of cell biology 91, 427-437 (1981).

86. Miner, J.H. et al. The Laminin $\alpha$ Chains: Expression, Developmental Transitions, and Chromosomal Locations of $\alpha 1-5$, Identification of Heterotrimeric Laminins 8-11, and Cloning of a Novel $\alpha 3$ Isoform. The Journal of cell biology 137, 685-701 (1997).

87. Sorokin, L.M., Pausch, F., Durbeej, M. \& Ekblom, P. Differential expression of five laminin alpha (1-5) chains in developing and adult mouse kidney. Developmental dynamics : an official publication of the American Association of Anatomists 210, 446-462 (1997).

88. Miner, J.H., Lewis, R.M. \& Sanes, J.R. Molecular cloning of a novel laminin chain, alpha 5, and widespread expression in adult mouse tissues. The Journal of biological chemistry 270, 28523-28526 (1995).

89. Zenker, M. et al. Human laminin beta2 deficiency causes congenital nephrosis with mesangial sclerosis and distinct eye abnormalities. Human molecular genetics 13, 2625-2632 (2004).

90. Miner, J.H. \& Li, C. Defective glomerulogenesis in the absence of laminin alpha5 demonstrates a developmental role for the kidney glomerular basement membrane. Dev Biol 217, 278-289 (2000).

91. Abrahamson, D.R., St John, P.L., Isom, K., Robert, B. \& Miner, J.H. Partial rescue of glomerular laminin alpha5 mutations by wild-type endothelia produce hybrid glomeruli. Journal of the American Society of Nephrology: JASN 18, 2285-2293 (2007).

92. Miner, J.H. \& Sanes, J.R. Collagen IV alpha 3, alpha 4, and alpha 5 chains in rodent basal laminae: sequence, distribution, association with laminins, and developmental switches. The Journal of cell biology 127, 879-891 (1994).

93. Miner, J.H. Developmental biology of glomerular basement membrane components. Curr Opin Nephrol Hypertens 7, 13-19 (1998).

94. Funk, S.D., Bayer, R.H. \& Miner, J.H. Endothelial Cell-Specific Collagen IV alpha3 Expression Does not Rescue Alport Syndrome in Col4a3-/- Mice. American journal of physiology. Renal physiology (2019).

95. Hudson, B.G., Tryggvason, K., Sundaramoorthy, M. \& Neilson, E.G. Alport's syndrome, Goodpasture's syndrome, and type IV collagen. N Engl J Med 348, 2543-2556 (2003).

96. Hudson, B.G. The molecular basis of Goodpasture and Alport syndromes: beacons for the discovery of the collagen IV family. J Am Soc Nephrol 15, 2514-2527 (2004).

97. Kalluri, R., Shield, C.F., Todd, P., Hudson, B.G. \& Neilson, E.G. Isoform switching of type IV collagen is developmentally arrested in X-linked Alport syndrome leading to increased susceptibility of renal basement membranes to endoproteolysis. Journal of Clinical Investigation 99, 2470-2478 (1997). 
98. Gunwar, S. et al. Glomerular basement membrane. Identification of a novel disulfide-crosslinked network of alpha3, alpha4, and alpha5 chains of type IV collagen and its implications for the pathogenesis of Alport syndrome. The Journal of biological chemistry 273, 8767-8775 (1998).

99. Gardner, H., Kreidberg, J., Koteliansky, V. \& Jaenisch, R. Deletion of integrin alpha 1 by homologous recombination permits normal murine development but gives rise to a specific deficit in cell adhesion. Dev Biol 175, 301-313 (1996).

100. Shrivastava, A. et al. An orphan receptor tyrosine kinase family whose members serve as nonintegrin collagen receptors. Molecular cell 1, 25-34 (1997).

101. Vogel, W., Gish, G.D., Alves, F. \& Pawson, T. The discoidin domain receptor tyrosine kinases are activated by collagen. Molecular cell 1, 13-23 (1997).

102. Reeves, W.H., Kanwar, Y.S. \& Farquhar, M.G. Assembly of the glomerular filtration surface. Differentiation of anionic sites in glomerular capillaries of newborn rat kidney. The Journal of cell biology 85, 735-753 (1980).

103. McCarthy, K.J., Abrahamson, D.R., Bynum, K.R., St John, P.L. \& Couchman, J.R. Basement membrane-specific chondroitin sulfate proteoglycan is abnormally associated with the glomerular capillary basement membrane of diabetic rats. The journal of histochemistry and cytochemistry : official journal of the Histochemistry Society 42, 473-484 (1994).

104. Karnovsky, M.J. \& Ainsworth, S.K. The structural basis of glomerular filtration. Advances in nephrology from the Necker Hospital 2, 35-60 (1972).

105. Rodewald, R. \& Karnovsky, M.J. Porous substructure of the glomerular slit diaphragm in the rat and mouse. The Journal of cell biology 60, 423-433 (1974).

106. Tojo, A. \& Endou, H. Intrarenal handling of proteins in rats using fractional micropuncture technique. The American journal of physiology 263, F601-606 (1992).

107. Russo, L.M. et al. The normal kidney filters nephrotic levels of albumin retrieved by proximal tubule cells: retrieval is disrupted in nephrotic states. Kidney international 71, 504-513 (2007).

108. Park, C.H. \& Maack, T. Albumin absorption and catabolism by isolated perfused proximal convoluted tubules of the rabbit. The Journal of clinical investigation 73, 767-777 (1984).

109. Dane, M.J. et al. Glomerular endothelial surface layer acts as a barrier against albumin filtration. The American journal of pathology 182, 1532-1540 (2013).

110. Jeansson, M. \& Haraldsson, B. Morphological and functional evidence for an important role of the endothelial cell glycocalyx in the glomerular barrier. American journal of physiology. Renal physiology 290, F111-116 (2006).

111. Friden, V. et al. The glomerular endothelial cell coat is essential for glomerular filtration. Kidney international 79, 1322-1330 (2011).

112. Smithies, O. Why the kidney glomerulus does not clog: a gel permeation/diffusion hypothesis of renal function. Proceedings of the National Academy of Sciences of the United States of America 100, 4108-4113 (2003).

113. Ogston, A.G. The spaces in a uniform random suspension of fibres. Transactions of the Faraday Society (1958).

114. Lawrence, M.G. et al. Permeation of macromolecules into the renal glomerular basement membrane and capture by the tubules. Proceedings of the National Academy of Sciences of the United States of America 114, 2958-2963 (2017).

115. Kestila, M. et al. Positionally cloned gene for a novel glomerular protein--nephrin--is mutated in congenital nephrotic syndrome. Molecular cell 1, 575-582 (1998).

116. Putaala, H., Soininen, R., Kilpelainen, P., Wartiovaara, J. \& Tryggvason, K. The murine nephrin gene is specifically expressed in kidney, brain and pancreas: inactivation of the gene leads to massive proteinuria and neonatal death. Human molecular genetics 10, 1-8 (2001).

117. Fissell, W.H. \& Miner, J.H. What Is the Glomerular Ultrafiltration Barrier? Journal of the American Society of Nephrology : JASN 29, 2262-2264 (2018).

118. Butt, L. et al. A molecular mechanism explaining albuminuria in kidney disease. Nature Metabolism 2, 461-474 (2020).

119. van den Hoven, M.J. et al. Reduction of anionic sites in the glomerular basement membrane by heparanase does not lead to proteinuria. Kidney international 73, 278-287 (2008).

120. Khalil, R. et al. Glomerular permeability is not affected by heparan sulfate glycosaminoglycan deficiency in zebrafish embryos. American journal of physiology. Renal physiology 317, F1211f1216 (2019).

121. Brenner, B.M., Hostetter, T.H. \& Humes, H.D. Molecular basis of proteinuria of glomerular origin. The New England journal of medicine 298, 826-833 (1978). 
122. Savige, J. et al. Expert guidelines for the management of Alport syndrome and thin basement membrane nephropathy. J Am Soc Nephrol 24, 364-375 (2013).

123. Miner, J.H. \& Sanes, J.R. Molecular and functional defects in kidneys of mice lacking collagen alpha 3(IV): implications for Alport syndrome. The Journal of cell biology 135, 1403-1413 (1996).

124. Rumpelt, H.J. Alport's syndrome: specificity and pathogenesis of glomerular basement membrane alterations. Pediatr Nephrol 1, $422-427$ (1987).

125. Khoshnoodi, J., Pedchenko, V. \& Hudson, B.G. Mammalian collagen IV. Microsc Res Tech 71, 357-370 (2008).

126. Kalluri, R., Shield, C.F., Todd, P., Hudson, B.G. \& Neilson, E.G. Isoform switching of type IV collagen is developmentally arrested in X-linked Alport syndrome leading to increased susceptibility of renal basement membranes to endoproteolysis. J Clin Invest 99, 2470-2478 (1997).

127. Funk, S.D., Lin, M.H. \& Miner, J.H. Alport syndrome and Pierson syndrome: Diseases of the glomerular basement membrane. Matrix Biol 71-72, 250-261 (2018).

128. Rubel, D. et al. Collagen receptors integrin alpha2beta1 and discoidin domain receptor 1 regulate maturation of the glomerular basement membrane and loss of integrin alpha2beta1 delays kidney fibrosis in COL4A3 knockout mice. Matrix Biol 34, 13-21 (2014).

129. Gross, O. et al. Loss of collagen-receptor DDR1 delays renal fibrosis in hereditary type IV collagen disease. Matrix Biol 29, 346-356 (2010).

130. Randles, M.J. et al. Basement membrane ligands initiate distinct signalling networks to direct cell shape. Matrix Biol (2020).

131. Abrahamson, D.R. et al. Laminin compensation in collagen alpha3(IV) knockout (Alport) glomeruli contributes to permeability defects. J Am Soc Nephrol 18, 2465-2472 (2007).

132. Cosgrove, D. et al. Integrin alphalbeta1 and transforming growth factor-betal play distinct roles in alport glomerular pathogenesis and serve as dual targets for metabolic therapy. Am J Pathol 157, 1649-1659 (2000).

133. Nozu, K. et al. A review of clinical characteristics and genetic backgrounds in Alport syndrome. Clin Exp Nephrol 23, 158-168 (2019).

134. Tryggvason, K. \& Patrakka, J. Thin basement membrane nephropathy. J Am Soc Nephrol 17, 813-822 (2006).

135. Savige, J. et al. Thin basement membrane nephropathy. Kidney Int 64, 1169-1178 (2003).

136. Zenker, M. et al. Human laminin beta2 deficiency causes congenital nephrosis with mesangial sclerosis and distinct eye abnormalities. Human molecular genetics 13, 2625-2632 (2004).

137. Noakes, P.G. et al. The renal glomerulus of mice lacking s-laminin/laminin beta 2: nephrosis despite molecular compensation by laminin beta 1. Nat Genet 10, 400-406 (1995).

138. Suh, J.H. \& Miner, J.H. The glomerular basement membrane as a barrier to albumin. Nat Rev Nephrol 9, 470-477 (2013).

139. Jarad, G., Cunningham, J., Shaw, A.S. \& Miner, J.H. Proteinuria precedes podocyte abnormalities inLamb2-/- mice, implicating the glomerular basement membrane as an albumin barrier. The Journal of clinical investigation 116, 2272-2279 (2006).

140. Ota, Z., Shikata, K. \& Ota, K. Nephrotic tunnels in glomerular basement membrane as revealed by a new electron microscopic method. J Am Soc Nephrol 4, 1965-1973 (1994).

141. Suh, J.H., Jarad, G., VanDeVoorde, R.G. \& Miner, J.H. Forced expression of laminin beta1 in podocytes prevents nephrotic syndrome in mice lacking laminin beta2, a model for Pierson syndrome. Proc Natl Acad Sci U S A 108, 15348-15353 (2011).

142. Saus, J., Wieslander, J., Langeveld, J.P., Quinones, S. \& Hudson, B.G. Identification of the Goodpasture antigen as the alpha 3(IV) chain of collagen IV. J Biol Chem 263, 13374-13380 (1988).

143. Foster, M.H. Basement membranes and autoimmune diseases. Matrix Biol 57-58, 149-168 (2017).

144. Fischer, E.G. \& Lager, D.J. Anti-glomerular basement membrane glomerulonephritis: a morphologic study of 80 cases. Am J Clin Pathol 125, 445-450 (2006).

145. McAdoo, S.P. \& Pusey, C.D. Anti-Glomerular Basement Membrane Disease. Clin J Am Soc Nephrol 12, 1162-1172 (2017).

146. Couser, W.G. Primary Membranous Nephropathy. Clin J Am Soc Nephrol 12, 983-997 (2017).

147. Liu, W. et al. Immunological Pathogenesis of Membranous Nephropathy: Focus on PLA2R1 and Its Role. Front Immunol 10, 1809 (2019).

148. Nangaku, M., Shankland, S.J. \& Couser, W.G. Cellular response to injury in membranous nephropathy. J Am Soc Nephrol 16, 1195-1204 (2005). 
149. van den Born, J. et al. Distribution of GBM heparan sulfate proteoglycan core protein and side chains in human glomerular diseases. Kidney Int 43, 454-463 (1993).

150. Raats, C.J. et al. Reduction in glomerular heparan sulfate correlates with complement deposition and albuminuria in active Heymann nephritis. J Am Soc Nephrol 10, 1689-1699 (1999).

151. Borza, D.B. Glomerular basement membrane heparan sulfate in health and disease: A regulator of local complement activation. Matrix Biol 57-58, 299-310 (2017).

152. Tervaert, T.W. et al. Pathologic classification of diabetic nephropathy. J Am Soc Nephrol 21, 556-563 (2010).

153. Kriz, W. et al. Accumulation of worn-out GBM material substantially contributes to mesangial matrix expansion in diabetic nephropathy. Am J Physiol Renal Physiol 312, F1101-F1111 (2017).

154. Kolset, S.O., Reinholt, F.P. \& Jenssen, T. Diabetic nephropathy and extracellular matrix. $J$ Histochem Cytochem 60, 976-986 (2012).

155. Mason, R.M. \& Wahab, N.A. Extracellular matrix metabolism in diabetic nephropathy. $J \mathrm{Am}$ Soc Nephrol 14, 1358-1373 (2003).

156. Stefan, G. et al. Histologic predictors of renal outcome in diabetic nephropathy: Beyond renal pathology society classification. Medicine (Baltimore) 98, e16333 (2019).

157. Conti, S. et al. Early and late scanning electron microscopy findings in diabetic kidney disease. Sci Rep 8, 4909 (2018).

158. Xu, X. et al. A glimpse of matrix metalloproteinases in diabetic nephropathy. Curr Med Chem 21, 3244-3260 (2014).

159. Li, Y. et al. C-peptide prevents SMAD3 binding to alpha promoters to inhibit collagen type IV synthesis. J Mol Endocrinol 61, 47-56 (2018).

160. Geng, X.D. et al. Identification of key genes and pathways in diabetic nephropathy by bioinformatics analysis. J Diabetes Investig 10, 972-984 (2019).

161. Kanwar, Y.S., Rosenzweig, L.J., Linker, A. \& Jakubowski, M.L. Decreased de novo synthesis of glomerular proteoglycans in diabetes: biochemical and autoradiographic evidence. Proc Natl Acad Sci U S A 80, 2272-2275 (1983).

162. Chetyrkin, S. et al. Glucose autoxidation induces functional damage to proteins via modification of critical arginine residues. Biochemistry 50, 6102-6112 (2011).

163. Voziyan, P., Brown, K.L., Chetyrkin, S. \& Hudson, B. Site-specific AGE modifications in the extracellular matrix: a role for glyoxal in protein damage in diabetes. Clin Chem Lab Med 52, 39-45 (2014).

164. Gautieri, A., Redaelli, A., Buehler, M.J. \& Vesentini, S. Age- and diabetes-related nonenzymatic crosslinks in collagen fibrils: candidate amino acids involved in Advanced Glycation End-products. Matrix Biol 34, 89-95 (2014).

165. Cole, J.B. \& Florez, J.C. Genetics of diabetes mellitus and diabetes complications. Nat Rev Nephrol (2020).

166. Guan, M. et al. Association of kidney structure-related gene variants with type 2 diabetesattributed end-stage kidney disease in African Americans. Hum Genet 135, 1251-1262 (2016).

167. Salem, R.M. et al. Genome-Wide Association Study of Diabetic Kidney Disease Highlights Biology Involved in Glomerular Basement Membrane Collagen. J Am Soc Nephrol 30, 20002016 (2019).

168. Miner, J.H. Type IV collagen and diabetic kidney disease. Nat Rev Nephrol 16, 3-4 (2020).

169. (US National Library of Medicine. ClinicalTrials.gov. https://clinicaltrials.gov/ct2/show/NCT02855268; 2020).

170. Wang, D. et al. The Chemical Chaperone, PBA, Reduces ER Stress and Autophagy and Increases Collagen IV $\alpha 5$ Expression in Cultured Fibroblasts From Men With X-Linked Alport Syndrome and Missense Mutations. Kidney Int Rep 2, 739-748 (2017).

171. Matsui, T. et al. RAGE-Aptamer Blocks the Development and Progression of Experimental Diabetic Nephropathy. Diabetes 66, 1683-1695 (2017).

172. Heidet, L. et al. A human-mouse chimera of the alpha3alpha4alpha5(IV) collagen protomer rescues the renal phenotype in Col4a3-/- Alport mice. Am J Pathol 163, 1633-1644 (2003).

173. Lin, X., Suh, J.H., Go, G. \& Miner, J.H. Feasibility of repairing glomerular basement membrane defects in Alport syndrome. J Am Soc Nephrol 25, 687-692 (2014).

174. Daga, S. et al. New frontiers to cure Alport syndrome: COL4A3 and COL4A5 gene editing in podocyte-lineage cells. Eur J Hum Genet (2019).

175. Ramsbottom, S.A. et al. Targeted exon skipping of a. Proc Natl Acad Sci U S A 115, 1248912494 (2018). 
176. Yamamura, T. et al. Development of an exon skipping therapy for X-linked Alport syndrome with truncating variants in COL4A5. Nat Commun 11, 2777 (2020).

177. McKee, K.K., Aleksandrova, M. \& Yurchenco, P.D. Chimeric protein identification of dystrophic, Pierson and other laminin polymerization residues. Matrix Biol 67, 32-46 (2018).

178. Walker, F. Basement-membrane turnover in man. The Journal of pathology 107, 123-125 (1972).

179. Adam Pickard, A.A., Yinhui Lu, Joan Chang, Richa Garva, Nigel Hodson, Karl E. Kadler Collagen assembly and turnover imaged with a CRISPR-Cas9 engineered Dendra2 tag. biorxiv (2018).

180. Gross, O. et al. Early angiotensin-converting enzyme inhibition in Alport syndrome delays renal failure and improves life expectancy. Kidney Int 81, 494-501 (2012).

181. Gomez, I.G. et al. Anti-microRNA-21 oligonucleotides prevent Alport nephropathy progression by stimulating metabolic pathways. J Clin Invest 125, 141-156 (2015).

182. Richter, H. et al. DNA-Encoded Library-Derived DDR1 Inhibitor Prevents Fibrosis and Renal Function Loss in a Genetic Mouse Model of Alport Syndrome. ACS Chem Biol 14, 37-49 (2019).

183. Aoki, Y. et al. Highly efficient in vivo delivery of PMO into regenerating myotubes and rescue in laminin- $\alpha 2$ chain-null congenital muscular dystrophy mice. Hum Mol Genet 22, 4914-4928 (2013).

\section{Acknowledgements}

This work was supported by a Wellcome Trust Senior Fellowship awarded (202860/Z/16/Z) to R.L. and supporting R.W.N., and by a FAPESP fellowship (2015/03525-2; 2017/26785-5) awarded to M.R.P.T.M.

\section{Author contributions}

The authors contributed equally to all aspects of the article.

\section{Competing interests}

The authors declare no competing interests.

\section{Peer review information}

Nature Reviews Nephrology thanks the anonymous reviewers for their contribution to the peer review of this work.

\section{Publisher's note}

Springer Nature remains neutral with regard to jurisdictional claims in published maps and institutional affiliations.

\section{Key Points}

- The glomerular basement membrane (GBM) is composed of many unique components that are likely to be important for appropriate GBM function. 
- The application of new imaging and proteomics technologies is enabling greater insight into GBM organisation in health and disease.

- The composition of the GBM changes during glomerulogenesis to permit proper development and filtration function.

- The GBM is a major contributor to the size-selectivity of the glomerular filter.

- Causes of GBM-associated disease include primary genetic defects in basement membrane components, and damage secondary to autoimmune and metabolic diseases, leading to abnormal synthesis and/or turnover of GBM proteins.

- Novel pharmacological and genome editing approaches might facilitate basement membrane repair and treatment of GBM-associated disease. 
Table 1 Current and investigative therapies for diseases with GBM involvement

\begin{tabular}{|c|c|c|c|c|}
\hline Target & Type & Mechanism of action & $\begin{array}{l}\text { Development } \\
\text { stage }\end{array}$ & References \\
\hline \multicolumn{5}{|c|}{ Available therapies } \\
\hline RAAS & $\begin{array}{l}\text { RAAS inhibitors } \\
\text { (ARBs, } \\
\text { aldosterone } \\
\text { blockers) }\end{array}$ & $\begin{array}{l}\text { Reduction in } \\
\text { intraglomerular } \\
\text { pressure }\end{array}$ & $\begin{array}{l}\text { Current } \\
\text { standard of care }\end{array}$ & Gross et al ${ }^{180}$ \\
\hline \multicolumn{5}{|c|}{ Therapies under development } \\
\hline \multirow[t]{3}{*}{ Fibrosis } & $\begin{array}{l}\text { Anti-miR-21 } \\
\text { oligonucleotide }\end{array}$ & $\begin{array}{l}\text { Reduces fibrosis in } \\
\text { Alport syndrome by } \\
\text { silencing miR-21 }\end{array}$ & $\begin{array}{l}\text { Preclinical study } \\
\text { (Col4a3 }^{-/-} \\
\text {mouse model of } \\
\text { Alport } \\
\text { syndrome); } \\
\text { clinical trial } \\
\text { ongoing }\end{array}$ & $\begin{array}{l}\text { Gomez et } \\
\text { al. }{ }^{181 ; N C T 02855268}\end{array}$ \\
\hline & $\begin{array}{l}\text { Collagen IV } \\
\text { receptor } \\
\text { inhibitors }\end{array}$ & $\begin{array}{l}\text { DDR1 selective } \\
\text { inhibitor; reduces } \\
\text { DDR1 phosphorylation }\end{array}$ & 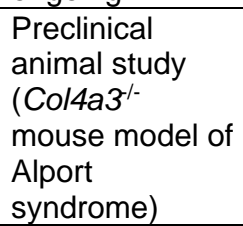 & Richter et al. ${ }^{182}$ \\
\hline & RAGE blocker & $\begin{array}{l}\text { Single-stranded DNA } \\
\text { aptamer against } \\
\text { RAGE; blocks AGE- } \\
\text { RAGE-signalling, } \\
\text { oxidative stress, } \\
\text { inflammation and ECM } \\
\text { accumulation }\end{array}$ & $\begin{array}{l}\text { Rat model of } \\
\text { diabetic kidney } \\
\text { disease } \\
\text { (induced with } \\
\text { streptozotocin) }\end{array}$ & Matsui et al. ${ }^{171}$ \\
\hline \multirow[t]{2}{*}{ ECM biology } & $\begin{array}{l}\text { Gene } \\
\text { replacement } \\
\text { therapy }\end{array}$ & $\begin{array}{l}\text { Inducible expression of } \\
\text { GBM genes }\end{array}$ & $\begin{array}{l}\text { Preclinical } \\
\text { animal study } \\
\text { (Col4a3 } \\
\text { mouse model of } \\
\text { Alport } \\
\text { syndrome) }\end{array}$ & Lin et al..$^{173}$ \\
\hline & $\begin{array}{l}\text { Chemical } \\
\text { chaperone- } \\
\text { based therapy }\end{array}$ & $\begin{array}{l}\text { Sodium 4- } \\
\text { phenylbutyrate; } \\
\text { increases COL4A5 } \\
\text { mRNA levels and } \\
\text { enhances the } \\
\text { deposition of COL4A5 }\end{array}$ & $\begin{array}{l}\text { Preclinical in } \\
\text { vitro study } \\
\text { (fibroblasts from } \\
\text { XLAS male } \\
\text { patient) }\end{array}$ & Wang et al. ${ }^{170}$ \\
\hline
\end{tabular}

AGE, advanced glycation endproducts; ARBs, angiotensin-receptor blockers; DDR1, discoidin domain receptor type-1; ECM, extracellular matrix; GBM, glomerular basement membrane; RAAS, renin-angiotensin-aldosterone system; RAGE, receptor for advanced glycation endproducts; XLAS, X-linked Alport syndrome. 
Figure 1. Major components of the glomerular basement membrane. The major components of all basement membranes include laminins, collagen IV, nidogens and the heparan sulphate proteoglycans agrin, perlecan and collagen XVIII. The GBM is enriched in the $\alpha 3 \alpha 4 \alpha 5$ isoform of collagen IV and also laminin-521, which interacts with cell surface receptors integrin $\alpha 3 \beta 1$ and $\alpha$-dystroglycan on podocytes and endothelial cells. Minor components such as fibulin-1 are also present in the GBM.

Figure 2. Glomerular matrix compartments and transition of basement membrane components during glomerular basement membrane development. a | The adult glomerulus contains three major distinct matrix compartments (red): the mesangial matrix, which is synthesized by mesangial cells to support the capillary loops; the glomerular basement membrane (GBM), which is synthesized by podocytes and endothelial cells; and the capsule matrix, which synthesized by the parietal cells of the Bowman's capsule. b | The composition of the GBM changes throughout development, with a switch in the composition of early laminin heterotrimers and the collagen IV network, coinciding with maturation of podocytes and the endothelium. col(IV), collagen IV; EC, endothelial cell.

Figure 3. Major and minor components of the glomerular basement membrane. An interactome - that is, a protein-protein interaction network - of the major and minor components within the glomerular matrix of human and mouse can be generated with use of mass spectrometry-based proteomics. Major components include the wellknown matrix proteins (for example, collagen IV and laminins), minor components include proteins more recently identified in the glomerular matrix (e.g., TINAG) and/or expressed during glomerular development (for example, LAMA1 and LAMB1). The interactome shown here was constructed using STRING v11. Each node represents a protein and connecting gray lines illustrate an interaction between connected proteins that has been experimentally verified and curated from protein interaction databases.

Figure 4. Morphological defects of the glomerular basement membrane associated with disease. a | The glomerular filtration barrier at the glomerular capillary wall is composed of interdigitating podocyte foot processes, the protein bridges of which form the slit diaphragm, and a fenestrated endothelial cell (EC) layer, separated by a thick central glomerular basement membrane (GBM). The composition and arrangement of these components are affected in numerous glomerular diseases. b| In Alport syndrome, the GBM is irregularly thickened and split or lamellated. 
Extensive podocyte foot process effacement is observed along with frequent podocyte protrusions invading the GBM. c | In thin basement membrane nephropathy is characterized by an abnormally thin GBM, with some degree of podocyte foot process effacement. d | In anti-GBM disease, anti-collagen IV- $\alpha 3$ autoantibodies are typically found deposited along the thickened GBM. e | The dominant features of membranous nephropathy include the deposition of large electron-dense immune-complexes on the subepithelial surface of the GBM, which is extensively damaged and thickened, and effacement or detachment of podocytes from the capillary wall. $f \mid$ Advanced diabetic kidney disease is associated with extensive GBM thickening, podocyte foot process effacement, retraction and detachment from the GBM. In addition, GBM and podocytederived material is often incorporated into the expanding mesangial matrix.

Figure 5. New technologies to repair defective basement membranes: a | Genetic approaches to repair damaged basement membranes include gene editing using systems such as CRISPR-Cas9 and exon skipping, which can be induced by CRISPRCas9, or with splice-blocking antisense oligonucleotides. For example, antisense oligonucleotide probes have been successfully used to remove mutated exons and splice sites during splicing and yield truncated mRNA that is translated into functional truncated proteins that partially restore basement membranes ${ }^{183}$. b | Protein linkagebased repair uses engineered linker proteins (such as $\alpha 1 \mathrm{LNNd}$ and $\beta \mathrm{LNNd}$ and miniagrin) to restore basement membrane assembly, cell-matrix anchorage and signalling. For example, transgenic expression of $\alpha 1 \mathrm{LNNd}$ subunits in laminin $\alpha 2$-deficient mice (a model of LAMA2-related muscular dystrophy) can restore polymerization of nonassembling laminin by providing laminin $\alpha$ chains with $\mathrm{LN}$-domain binding sites to laminin $\beta$ and $\gamma$ chains. $c \mid$ Restorative protein therapy could also be used to replace missing basement membrane proteins. For example, injection of human recombinant laminin-521 (hLM-521) effectively replaced the missing laminin-521 network in the subendothelial aspect of the GBM in Lamb2 $2^{-/}$mice (a model of Pierson syndrome) and delayed the onset of proteinuria. 


\section{Glossary}

\section{Type IV collagen}

Encoded for by six genes in vertebrates (COL4A1-COL4A6), type IV collagen provides a scaffold and is present in basement membranes. Type IV collagen proteins form three heterotrimers $(\alpha 1 \alpha 1 \alpha 2, a 3 \alpha 4 \alpha 5, \alpha 5 a 5 a 6)$ that establish networks in the extracellular space.

\section{Nidogens}

Formerly known as entactin, nidogens are dumbbell-shaped proteins found in all basement membranes. Nidogen functions to connect collagens and laminins in the matrix.

\section{Heparan-sulphate proteoglycans}

(HSPGs). A large family of extracellular and membrane-attached molecules formed by a major protein that contains one or more covalently attached heparin sulphate glycosaminoglycan chains. Examples of HSPGs in basement membranes include collagen XVIII, agrin and perlecan.

\section{Collagen XVIII}

A heparin-sulphate proteoglycan that exists as a homotrimer in basement membranes. It has an important role in maintaining basement membrane integrity by mediating signalling (for example canonical Wnt signalling) and cell-matrix interactions (such as with integrin and VEGF receptors via a C-terminal endostatin domain).

\section{Laminin}

A family of glycoproteins that exist as heterotrimers with $\alpha, \beta$, and $y$ subunits. Laminin heterotrimers polymerise in the extracellular space to form a sheet that is essential for the formation and function of basement membranes.

\section{Plakins}

A family of large binding proteins that link cytoskeletal intermediate filaments to other filaments and to junctional complexes such as desmosomes and hemidesmosomes. 


\section{Serial block-face SEM}

(SBF-SEM). An imaging approach that uses a scanning electron microscope equipped with an automated ultramicrotome that sequentially cuts resin-embedded samples and scans the block surface in a repetitive manner to yield a stack of aligned images with transmission EM quality.. This approach provides volumetric imaging data of tissue structures in the $\mathrm{X}, \mathrm{Y}$ and $\mathrm{Z}$-axes.

\section{Low-vacuum SEM}

(LV-SEM). An EM approach that allows the scanning of non-conductive hydrous samples achieving EM resolution without prominent charging artifacts

\section{Helium ion microscopy}

(HIM). An EM approach in which the beam of electrons used for scanning and surface imaging in conventional SEM is replaced by a focused beam of helium ions to minimize sample damage and obtain high magnification images of uncoated soft specimens with improved sub-nanometer resolution, and high surface focus and contrast.

\section{Stimulated emission depletion}

(STED). A super resolution microscopy approach that uses a double laser beam to scan multiple fluorescent markers at the same time. The first beam stimulates fluorescence whilst a second creates a light annulus superimposed on top of the first to deplete fluorescence, creating a small scanning beam below the diffraction limit of resolution.

\section{Stochastic optical reconstruction microscopy}

(STORM). Super resolution microscopy approach that uses stochastic photoswitchable fluorescent probes that are activated individually by a weak light source to emit light separately for short time intervals to produce a high-resolution image constructed point-by-point based on the precise location of each individual fluorophore.

\section{Tissue expansion}

A sample preparation technique in which a tissue sample embedded within a polymer meshwork (for instance a hydrogel) is uniformly expanded to enable nanoscaleresolution imaging of preserved tissue structures by immunofluorescence staining and diffraction-limited microscopy.

\section{Haemangioblasts}


Multipotent precursor cells that can differentiate into endothelial cells and any cell type within the haematopoietic lineage.

\section{Microcoria}

A congenital disorder of the eye characterised by small pupils with a diameter less than $2 \mathrm{~mm}$.

\section{Advanced glycation end products}

(AGEs). Proteins or lipids that are modified by the non-enzymatic binding of a reactive sugar to basic amino acids such as lysine or arginine.

\section{Exon skipping therapy}

A technology that can be used to correct the coding reading frame of a mRNA transcript from a mutated gene in order to produce a functional protein.

\section{Joubert syndrome}

An inherited ciliopathy caused by mutations in more than 30 genes required for normal cilia function. 


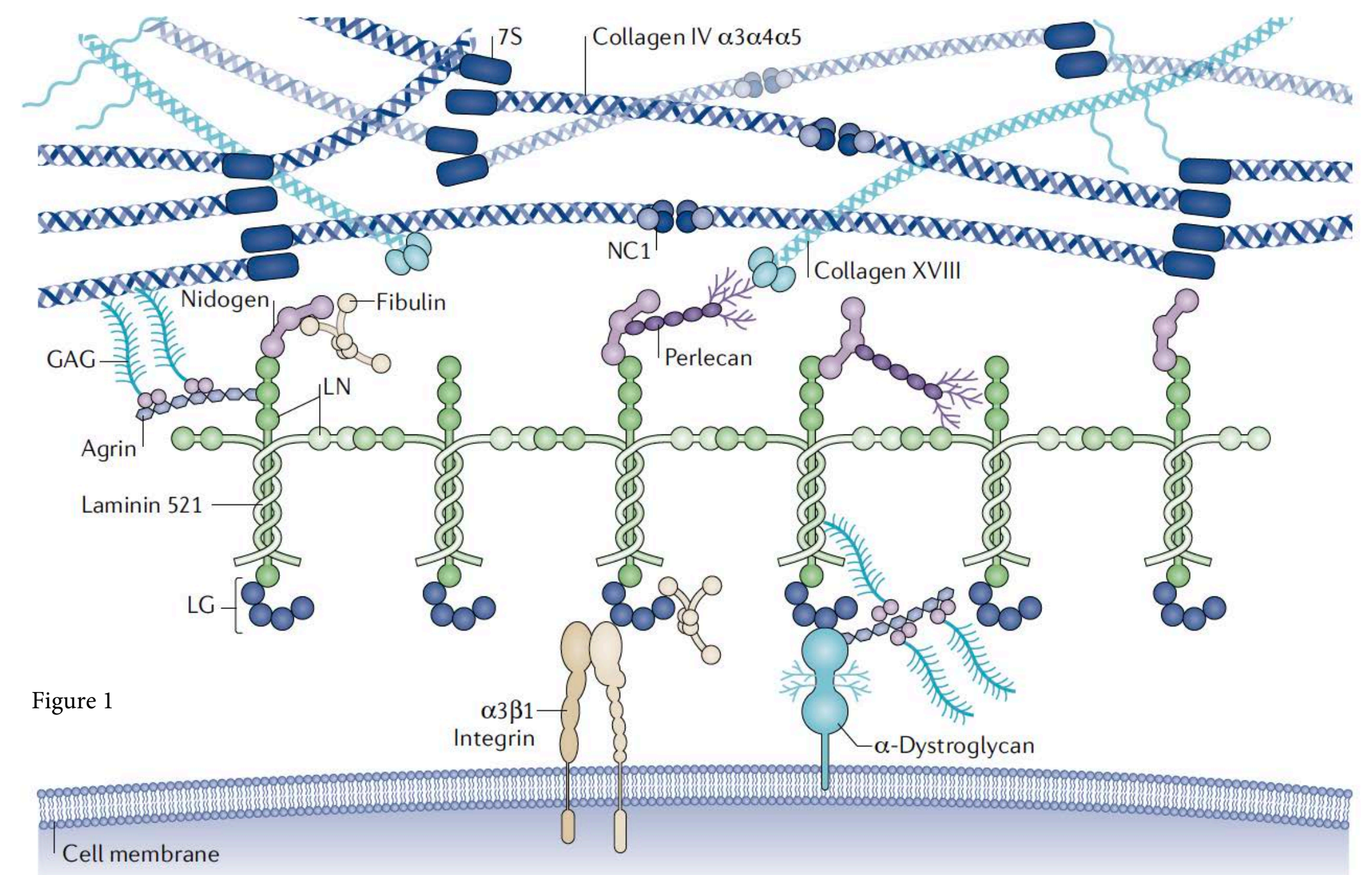




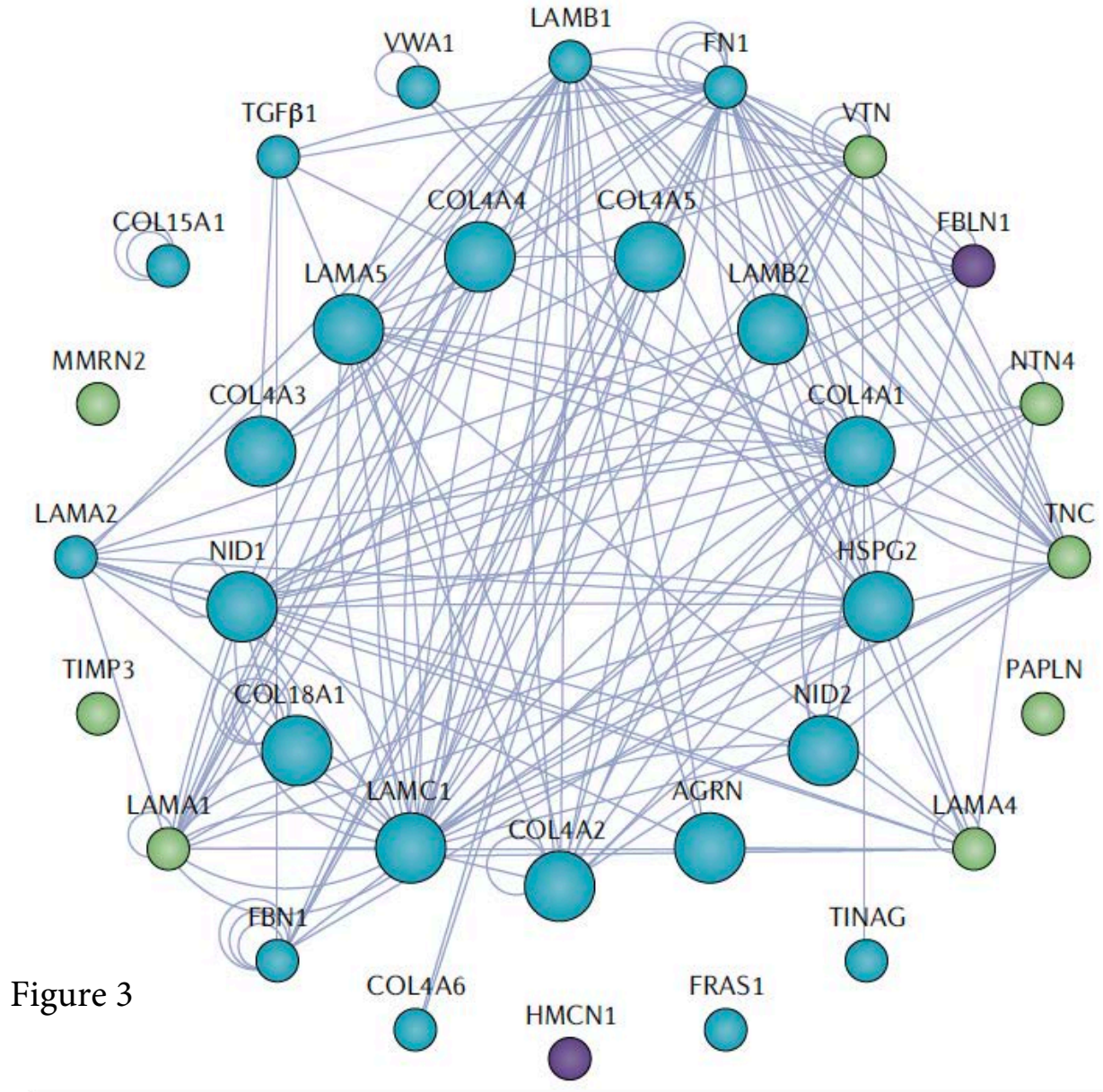

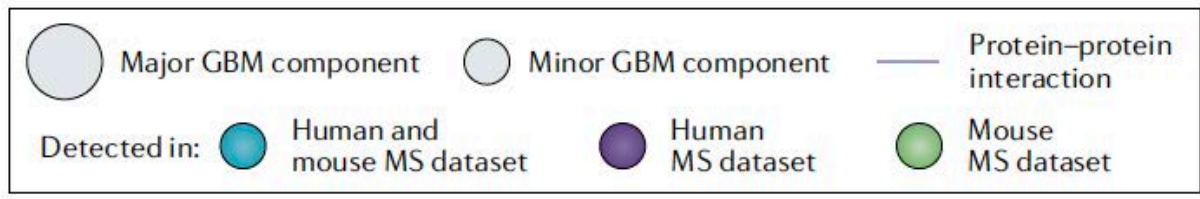



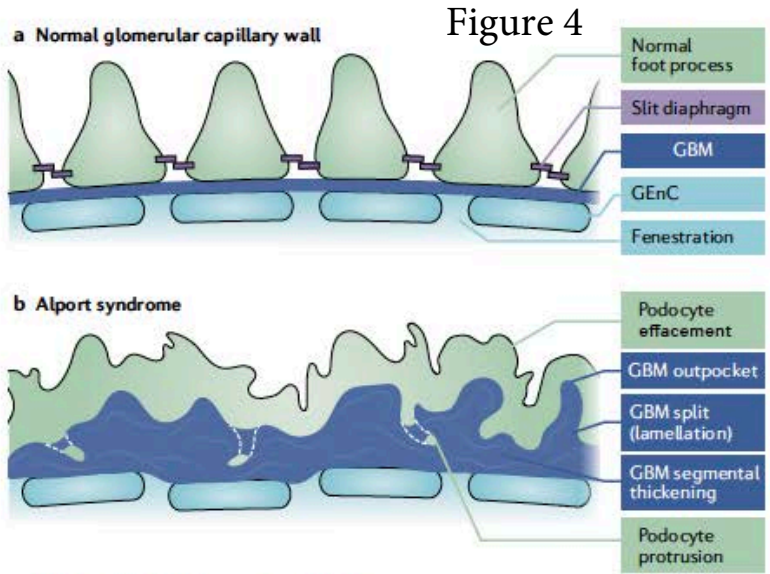

c Thin basement membrane nephropathy

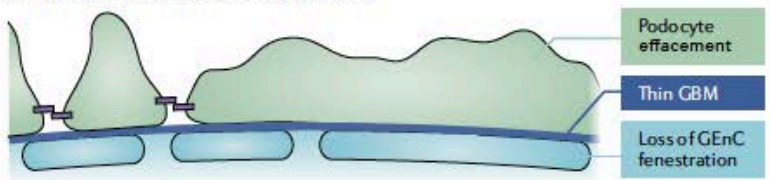

\section{d Anti-GBM glomerulonephrit is}

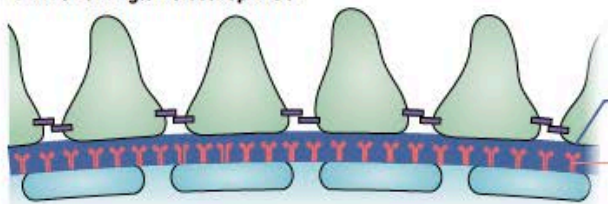

Thickened GBM

Layer of auto-

antibodies anticollagen- $\alpha 3(\mathrm{M})$

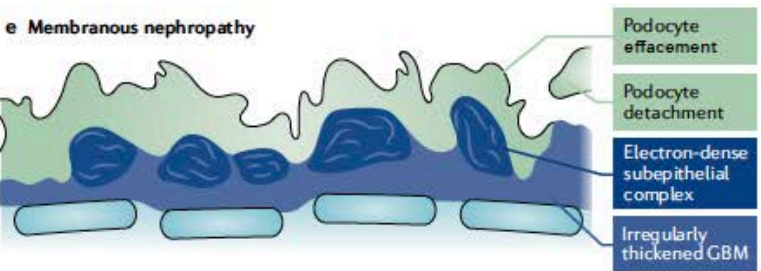

\section{f Diabetic kidney disease}

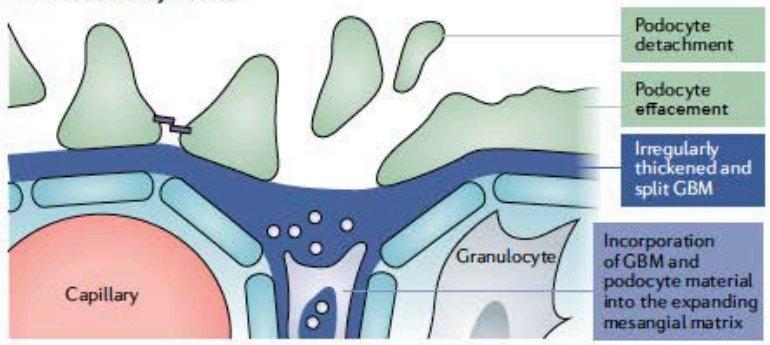


Figure 5

a Gene-editing technologies
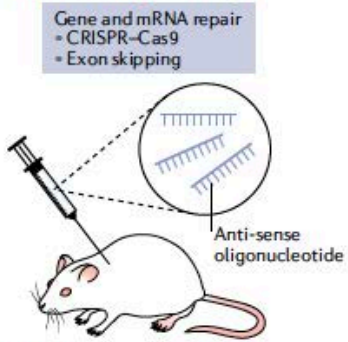

Mutated sequence

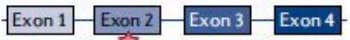

Intron 1 Intron 2 Intron 3

AO hybridization

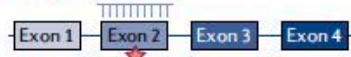

Intron 1 Intron 2 Intron 3

Truncated spliced

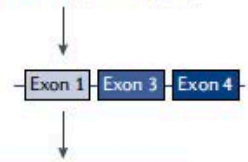

sequence

Functional truncated protein b Protein linkage-based repair

Linker proteins
- LNNd
- Mini-agrin

Laminin

$\alpha 2$-deficient

mouse

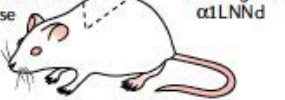

Non-assembling laminins and transgenic $\alpha 1$ LNNd

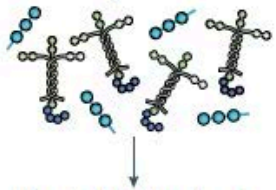

Repaired laminin network

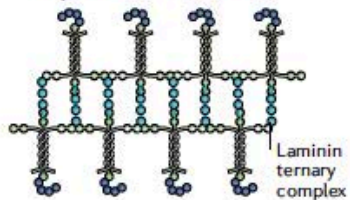

c Protein replacement

\section{Restorative} protein therapy

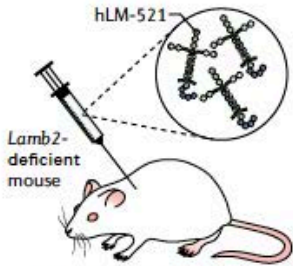

Missing LM-521 network

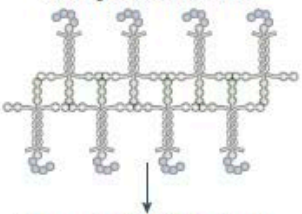

Transgenic hLM-521 network

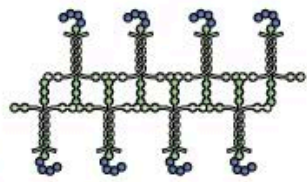

